\title{
Local perception of ecosystem services and their conservation in Sudanian savannas of Burkina Faso (West Africa)
}

\author{
Assétou Nabaloum ${ }^{1 *} \mathbb{D}$, Dethardt Goetze ${ }^{2}$, Amadé Ouédraogo $^{1}$, Stefan Porembski ${ }^{2}$ and Adjima Thiombiano ${ }^{1}$
}

\begin{abstract}
Context: In Burkina Faso, Sudanian savannas are important ecosystems for conservation of plant diversity. Due to desertification and insecurity, population migration from the North has increased human density and anthropogenic pressure on southern savannas. This study aims to investigate knowledge of local populations on ecosystem services (ES) and perception of their conservation.

Method: Individual semi-structured interviews about knowledge on ES and ecosystem conservation issues were conducted. Informants were selected according to sociocultural groups and sex in three areas of different land use intensity: the communal area of Dano (CAD), the Total Wildlife Reserve of Bontioli (TWRB) and the Game Ranch of Nazinga (GRN). The use value and vulnerability index of each plant species were determined. A cluster analysis and a principal component analysis were carried out to identify the particular knowledge of different ethnic groups.

Results: Overall, 163 plant species were cited for fifteen ES. Provisioning services were most frequently cited (100\%), regulating services second most frequently (92.47\%). Entire plants were exclusively used for ES with non-material benefits (protection against wind, for shading, soil fertility, erosion prevention, tourism and religion). The ten species contributing most to ES provision were Vitellaria paradoxa, Parkia biglobosa, Diospyros mespiliformis, Adansonia digitata, Lannea microcarpa, Faidherbia albida, Khaya senegalensis, Afzelia africana, Ficus sycomorus, Pterocarpus erinaceus. Seven of them were identified as highly vulnerable. Around GRN, migrants and natives shared the same knowledge, while migrants in TWRB used the ES only to a small extent due to restricted contact with the native population. Migrants and natives of GRN had more knowledge on tourism and crafts services while the natives of CAD and TWRB made use of the services that sustain the quality of the agricultural land and meet their primary needs. To reduce further degradation, different communities suggested unanimously raising awareness of the importance of biodiversity and ecosystem conservation. The most quoted motivations to preserve ecosystems were vegetation sustainability and village development.
\end{abstract}

Conclusion: This study documented important local knowledge-based information to guide cultivation of local multipurpose species and initiation of communities to practice best management strategies for sustainable conservation of biodiversity.

Keywords: Indigenous knowledge, Land use, Plant vulnerability, Sustainable management, Sociocultural groups

*Correspondence: asnab2000@gmail.com

${ }^{1}$ Laboratory of Plant Biology and Ecology, University Joseph Ki-Zerbo, 03 BP 7021 Ouagadougou 03, Burkina Faso

Full list of author information is available at the end of the article

\section{Background}

Ecosystem services (ES) are defined as the goods and services obtained by the human population from ecosystems, directly or indirectly, to assure its well-being [1]. ES 
can be classified in four main categories. The supporting services derive from general functioning of an ecosystem, the regulating services correspond to the direct services of ecological functions on site, and provisioning and cultural services refer to direct services of obtaining goods and social and spiritual well-being from ecosystems. While the provisioning services provide finished products of ecosystems, the non-material cultural services allow for developing and enriching knowledge systems, social relationships and aesthetic values [2]. Products of ecosystems used for provisioning services (such as food, fodder, wood, medicinal compounds) include non-timber forest products (NTFP) such as fruits, leaves, seeds, flowers, bark, medicinal herbs, as well as wood cut from trees for supply of energy and for construction.

In West African semi-arid areas, local populations strongly depend on plant resources for meeting their daily needs [3-6]. Local people consider savanna ecosystems as their own good, as granary, pharmacy, pasture, place of religious worship and source of the strength of their territory [7]. Ecosystem functions and services do not only result from good ecosystem health, but also from the use that populations have made in various biogeographic and geo-economic contexts [8].

In Sudanian savanna ecosystems, climate and soil characteristics are favorable to the development of a diversified and dense vegetation cover [9]. In the semi-arid context of Burkina Faso, Sudanian savannas constitute a particular hot spot of plant species diversity $[10,11]$. Here, Zizka et al. [10] recorded $71 \%$ of all plant species of the country, with more than half of them being rare. Recent migration of human populations from the North to the South, fleeing desertification and climate change consequences on arable lands, has caused population density to increase in Sudanian savannas. In addition, terrorist attacks in the northern and eastern regions of the country have caused a new wave of migration and a raise in population density from 27.3 to 51.66 inhabitants $/ \mathrm{km}^{2}$ in the Southwest region between 1985 and 2019 [12]. This population growth has immediate consequences such as intensification of anthropogenic pressure on plant resources and protected areas [13]. Dimobe et al. [14 and 15] noted a significant decrease in natural vegetation from 1984 to 2013, followed by an expansion of croplands and habitat fragmentation in protected areas in the South Sudanian zone of the country [15].

Inclusive sustainable management of plant resources by the forest authority in collaboration with local populations could be a response to the strong anthropogenic pressure that ecosystems are facing, as the fundamental ecological role of forests is as important as their economic and social roles to local populations [3]. Indigenous people play a crucial role in biodiversity conservation through their traditional knowledge on species and habitats and their socioeconomic and symbolic practices as well [16]. However, many local populations suffer from consequences of large development projects and exploitation of natural resources. Among these consequences are land expropriation, loss of identity, language and culture [16]. Thus, local populations adapt their use to the change they perceive [8] in different ecosystems. They know that their survival essentially depends on their adaptation to the socio-environmental impact of climate change [17]. Better conservation of ecosystems requires good mastering of endogenous and scientific knowledge [7]. In addition, together with processes of decentralization and population self-management, the local scale is the relevant geographic and socio-economic space for conducting participatory development policies [18]. According to Holou and Sinsin [19], it would allow for answering one of the major concerns of African countries which is the rational and sustainable management of natural resources.

In the last decade, research questions on ES have been increasingly focused on provisioning services [4, 5, 2025]. In order to design effective vegetation management that prevents damage and promotes well-being of local people, it is important to take into account the needs of the latter. Thus, the present study aims to (i) understand knowledge of local populations on ecosystem services provided by plant species, (ii) identify factors that influence this knowledge, and (iii) understand local perceptions of sustainable management of plant communities.

\section{Methods}

\section{Study area}

The study was carried out in the South Sudanian phytogeographical sector of Burkina Faso [9]. The sampling areas were chosen according to variations in land-use intensity and identified through classification of landuse/land cover data from multi-temporal Landsat images (for methodological details see [14, 15]), allowing for assigning them to a three-point scale with high, medium and low land-use intensity [26]. The communal area of Dano (CAD), located in the southwestern region of Burkina Faso and characterized by agrosystems and grazing land, corresponds to the high land-use intensity level (Fig. 1). The Total Wildlife Reserve of Bontioli (TWRB), also located in the southwestern region, consists of a protected area of IUCN category I under several human pressures, and corresponds to the medium land-use intensity level. The Game Ranch of Nazinga (GRN) and its ZOVICs (village hunting zones), which are protected areas of IUCN category VI, devoted to hunting and located in the South-central region, corresponds to the low land-use intensity level. 


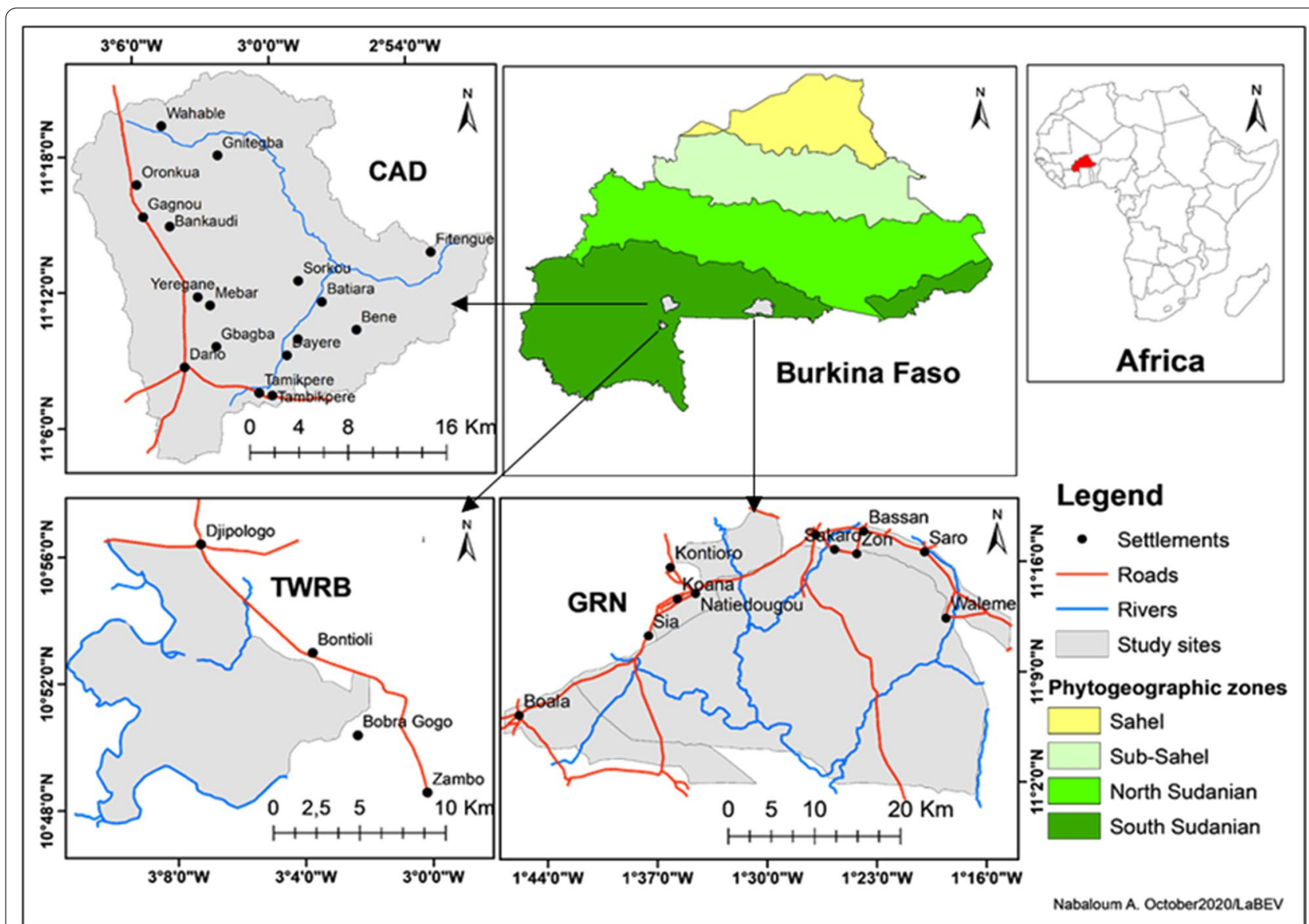

Fig. 1 Location of the study sites in Burkina Faso. TWRB: Total Wildlife Reserve of Bontioli (medium use intensity); CAD: communal area of Dano (high use intensity); GRN: Game Ranch of Nazinga (low use intensity)

The vegetation types in the study area are grass savannas, shrub savannas, tree savannas, savanna woodlands, woodland and gallery forests [27]. Dominant woody species are Vitellaria paradoxa C.F. Gaertn, Terminalia laxiflora Engl. \& Diels, Terminalia macroptera Guill. \& Perr., Combretum glutinosum Perr. ex DC., Combretum micranthum G. Don, Combretum adenogonium Steud. ex A.Rich., Combretum collinum Fresen., Anogeissus leiocarpa (DC.) Guill. \& Perr., Detarium microcarpum Guill. \& Perr., Piliostigma thonningii (Schum.) Milne-Redhead, Isoberlinia doka Craib \& Stapf and Lannea acida A. Rich. The dominant grass species are Andropogon gayanus Kunth, Hypparhenia rufa (Nees) Stapf, Loudetia togoensis (Pilger) C.E. Hubbard and Schizachyrium sanguineum (Retz.) Alston $[14,15]$.

The climate of the study area is Sudanian with a unimodal rainy season of 5 to 6 months from May to September or October. The mean annual rainfall during a 30 -year period (1986-2015) is $1048.73 \pm 146.7 \mathrm{~mm}$. The mean temperature for this same period is $28.1 \pm 2.15{ }^{\circ} \mathrm{C}$ (National Direction of Meteorology of Burkina Faso).
The native sociocultural groups are the Dagara and the Pougouli in CAD, the Dagara in TWRB and the Kassena in GRN. The dominant non-native sociocultural group encountered in all sites are the Mossi which have moved to these areas to practice agriculture on more fertile soils and recently for gold panning. Agriculture, livestock and gold panning constitute the main human activities in the study area.

\section{Sampling design and data collection Ethnobotanical data}

Seventeen villages were randomly selected in the three study sites following a random sampling scheme: seven villages in CAD, five around TWRB and five around GRN. The selection of informants was stratified, based on sociocultural groups (Dagara, Pougouli, Mossi, Kassena) and sex [28]. The Mossi were immigrants and the other ones indigenous (Table 1). Individual semi-structured interviews were conducted from December 2016 to March 2017 to collect the data following a questionnaire. In each village and for each sociocultural group, 
Table 1 Sociodemographic characteristics of the informants

\begin{tabular}{llll}
\hline Study sites & CAD & TWRB & GRN \\
\hline Number of villages & 7 & 5 & 5 \\
Sociocultural group & Dagara (71\%), & Dagara (71\%), & Kassena (50\%), \\
& Pougouli (29\%) & Mossi (29\%) & Mossi (50\%) \\
Gender & Male (50\%); & Male (50\%); & Male (50\%); \\
& Female (50\%) & Female (50\%) & Female (50\%) \\
Age classes & Young (34\%); & Young (31\%); & Young (42\%); \\
& Adult (42\%); & Adult (46\%); & Adult (38\%); \\
& Elder (24\%) & Elder (23\%) & Elder (20\%)
\end{tabular}

TWRB, Total Wildlife Reserve of Bontioli (medium use intensity); CAD, communal area of Dano (high use intensity); GRN, Game Ranch of Nazinga (low use intensity); Age classes, Young $=[20-40[$; Adult $=[40-60[;$ Elder $\geq 60$

ten informants (five men and five women were selected randomly) of at least 20 years of age were interviewed with their consent, yielding a total sample of 240 informants (Table 1). Interviews were conducted in the local language of the informants and translated by a local translator. Informants were asked to list the plant species they use and the ES rendered, and the used organs were recorded. Informants were also questioned about availability and dynamics of the plant species, reasons for these dynamics, solutions in case of regression and motivations to conserve biodiversity. Each informant classed the items of suggested solution and motivation by preference order. For identifying the plant species cited in local language, in each study site, the "walk-inthe woods" method was used at the end of the interviews. This method consisted in field visits with members of the community who have good knowledge about plant species [29]. They were selected for having cited the largest number of utilized species during the interview phase. During the field visit, fresh samples of cited species were collected and pressed for identification using the floras of Berhaut [30], Lebourgeois and Merlier [31], Poilecot [32] and the field handbook of Arbonnier [33]. The identified samples were verified by comparison with samples of the Ouagadougou herbarium at Joseph Ki-Zerbo University.

\section{Floristic data}

A vulnerability index was calculated taking into account species frequencies in the study area [20,34,35]. For this purpose, vegetation surveys were conducted in 152 plots distributed in different vegetation types (grass savannas, shrub savannas, tree savannas, savanna woodlands, woodland and gallery forest) and the fields throughout the study area. The sampling unit was a $50 \mathrm{~m} \times 20 \mathrm{~m}$ $(1000 \mathrm{~m} 2)$ in non-cultivated savannas, $50 \mathrm{~m} \times 10 \mathrm{~m}$ $(500 \mathrm{~m})$ in gallery forest, and $50 \mathrm{~m} \times 50 \mathrm{~m}(2500 \mathrm{~m} 2)$ in cultivated areas [36]. These plot dimensions are used to take into consideration the spatial distribution of most species with unevenly spread individuals [36].

\section{Data analyses}

Firstly, useful species cited by informants were ranked according to the fifteen most cited ES of the four categories of provisioning, regulating, cultural and supporting ES $[1,5]$. Ethnobotanical indices were calculated to assess the importance of services provided by each species. They are:

- the relative frequency of organ citation (RFO) with the adapted formula from Camou-Guerrero et al. [37] $\mathrm{RFO}=(\mathrm{Nuh} / \mathrm{Ntu}) \times 100$

where Nuh represents the number of citations of uses of the organ and Ntu the number of citations of all the organs in each ES;

- the relative frequency of service citation (RFS) $\mathrm{RFS}=(\mathrm{Nuh} / \mathrm{Ntu}) \times 100$

where Nuh represents the number of citations of one ES and Ntu the number of citations of all ES at one level of land use intensity.

- the actual UV index of a species (mean of the number of distinct actual uses reported per informant) $U V=\Sigma \mathrm{Ui} / U t$

where Ui denotes the number of different uses of a species and Ut the total number of people who cited the species.

Secondly, a nonparametric test of Kruskal-Wallis at the $5 \%$ threshold was carried out to compare the different ES quoted by the populations pertaining to age classes, gender, land use intensity and sociocultural groups, where $X^{2}$ represents the approximate value of modal distribution. The degree of freedom, D.f $=$ effectif -1 and a $P$ value 0.05 indicate a significant difference in results. The analyses were processed with $\mathrm{R}$ software [38], with etnobotanyR and agricolae packages. A cluster analysis was carried out to determine the degree of similarity between the knowledge of different sociocultural groups; afterward, a principal component analysis (PCA) was performed with PcOrd9 software to assess the links between sociocultural groups (individuals) and knowledge on ES (initial variables).

Thirdly, the conservation status of the ten most used species was determined by the vulnerability index calculation [34] which is the average of the highest values of seven selected parameters (Table 2). According to Betti [34], if $\mathrm{VI}<2$, the species is assumed to be weakly vulnerable, if $2 \leq \mathrm{VI}<2.5$, the species is moderately vulnerable, and if $\mathrm{VI} \geq 2.5$, the species is highly vulnerable. 
Table 2 Applied parameters of the vulnerability index

\begin{tabular}{|c|c|c|c|}
\hline Parameters & 1 (Low scale) & 2 (Average scale) & 3 (High scale) \\
\hline Use frequency (N1) & $N 1<20 \%$ & $20 \% \leq N 1<60 \%$ & $N 1 \geq 60 \%$ \\
\hline Number of uses (N2) & $\mathrm{N} 2<2$ & $2 \leq \mathrm{N} 2 \leq 4$ & $\mathrm{~N} 2 \geq 5$ \\
\hline Plant parts used (N3) & Leaves, latex & Fruits, branches & Wood, seeds, bark, roots, flowers \\
\hline Biotope of plant (N4) & Ruderal, gardens, field & Secondary forest & Primary or undisturbed forest \\
\hline Collection mode (N5) & Collection on the ground & & Collection on the tree, cutting \\
\hline Development stage (N6) & Old, senescent & Adult & Young \\
\hline $\begin{array}{l}\text { Relative frequency in the environment } \\
\text { (N7) }\end{array}$ & $\mathrm{Rf} \geq 2 / 3 \mathrm{Fm}$ & $1 / 3 \mathrm{Fm} \leq \mathrm{Rf}<2 / 3 \mathrm{Fm}$ & $\mathrm{Rf}<1 / 3 \mathrm{Fm}$ \\
\hline
\end{tabular}

Rf, relative frequency; Fm, maximum frequency

An adaptation of the method of Lawrence et al. [39] was used to assess the orders of preference of causes of degradation, recommended solutions, and motivations for ecosystem preservation. The ranks given by each informant were converted into scores. The used scores were grades decreasing from the number of items in each question. For example, if 7 causes were cited for ecosystem degradation, the used scores were scores starting from 7 and decreasing in the order of the informant's citation. When an item was not cited by an informant, a score of 0 was given. For each item, the average score was calculated for each socio-cultural group, age group, and gender.

$$
V t i=\sum T i / N i
$$

Vti: the average score given to a given item by a category of informants; Ti: the sum of the scores given to this item by this category of informants; Ni: the number of informants from this category of informants.

\section{Results}

Diversity of used plant species and ecosystem services

Major ES providers to local populations were 163 species including 130 woody species and 33 herbaceous species (belonging to 122 genera and 42 families). In all categories of land-use intensity, the most dominant families were Fabaceae (35 species), Poaceae (17 species), and Malvaceae (13 species). Each ES concerned a great diversity of plant species. At least 60 species were used for 10 ES. Provisioning services were accomplished with the highest number of species (Fig. 2) and were the most cited (67\%) by the informants. These services concerned medicinal use (120 species), fodder (76 species), and food supply (75 species). The species used in these three services had multipurpose uses. Diospyros mespiliformis, Khaya senegalensis, Vitellaria paradoxa and Saba senegalensis were quoted for $14 \mathrm{ES}$.

When considering the study area, the relative frequencies of number of citations varied significantly between the different ES $\left(\chi^{2}=1849.6 ; \quad \mathrm{Dl}=14 ; \quad P\right.$ value $<0.0001)$. By far the most quoted ES were food supply (25.98\%) and medicinal use (20.89\%), followed by fodder $(7.33 \%)$, craft $(6.10 \%)$ and energy supply (6.09\%), which are all provisioning services (Fig. 3). Protection against wind (6.08\%) and shading (6\%) (regulating services) were important services, too.

The relationships between ES and plant organs used for them (Fig. 4) showed that the entire plant was exclusively (100\%) cited for six ES, notably soil fertility (supporting service), religion, tourism (cultural services), and shading, protection against wind and erosion prevention (regulating services).

Wood was exploited for the three provisioning services energy supply (100\%), construction (100\%) and craft (65\%). Plant stalks were used only for craft and ceremony services. Plant leaves and fruits were cited for provisioning (food, fodder supply, medicinal use), regulating (pest control, water purification) and cultural (ceremonies) services with varying relative frequencies. Fruits were cited more often (70\%) for food service. Flowers and seeds were used for food only. The highest number of organs (5) was cited for medicinal services, including bark and roots.

The use values (UV) computed for all quoted species showed that many species were exploited for several services. Woody species were the plant category with the highest UV (i.e., the most used species). Vitellaria paradoxa $(\mathrm{UV}=3.775)$ was the species with the highest use value in the study sites. The three most used herbaceous species were Andropogon gayanus Kunth (UV=0.723), Rottboellia cochinchinensis (Lour.) Clayton (UV $=0.400)$ and Hyptis spicigera Lam $(U V=0.370)$. In general, the ten species with the highest use values were in decreasing order: Vitellaria paradoxa, Parkia biglobosa, Diospyros mespiliformis, Adansonia digitata, Lannea microcarpa, Faidherbia albida, Khaya senegalensis, Afzelia africana, Ficus sycomorus, and Pterocarpus erinaceus (Table 3 ). 


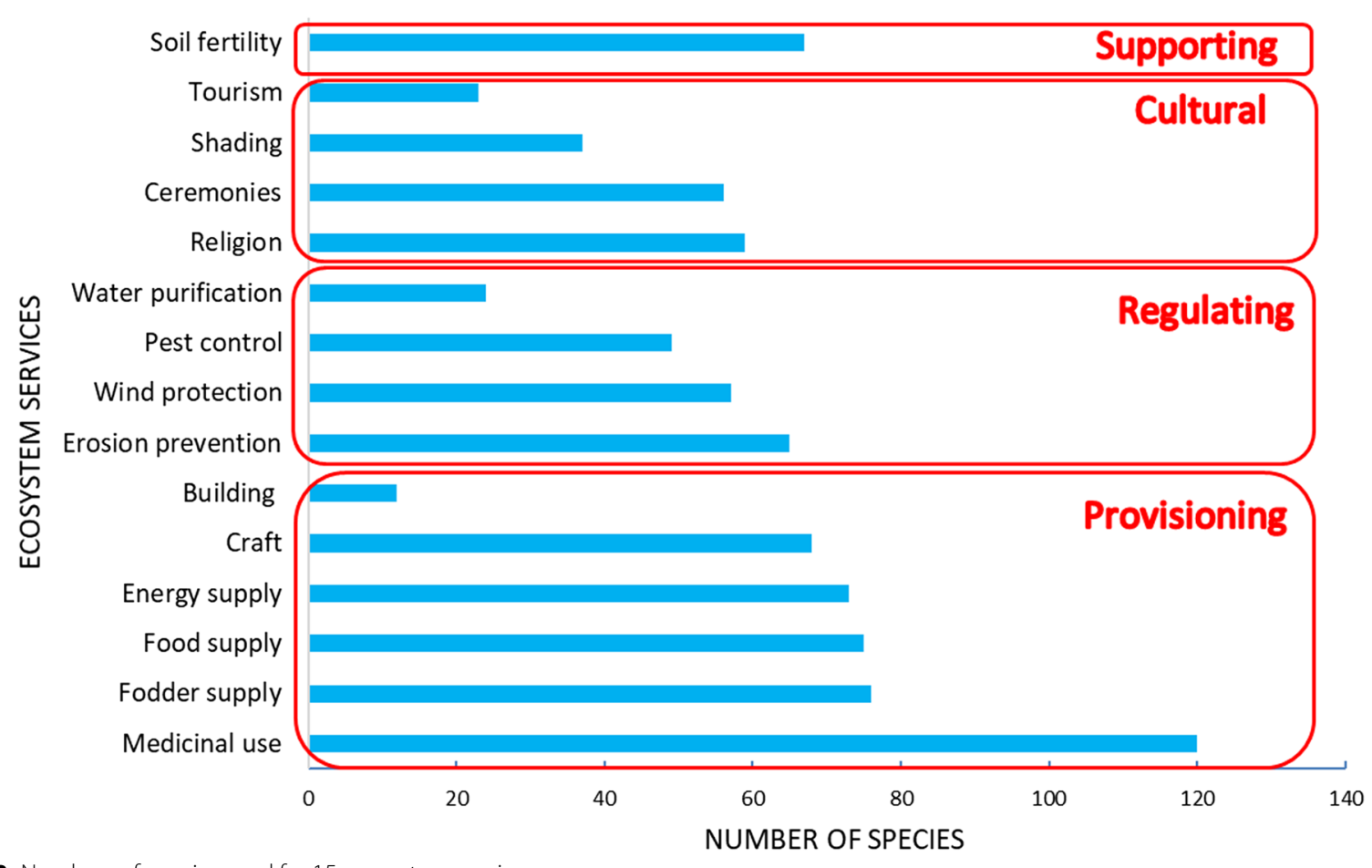

Fig. 2 Numbers of species used for 15 ecosystem services

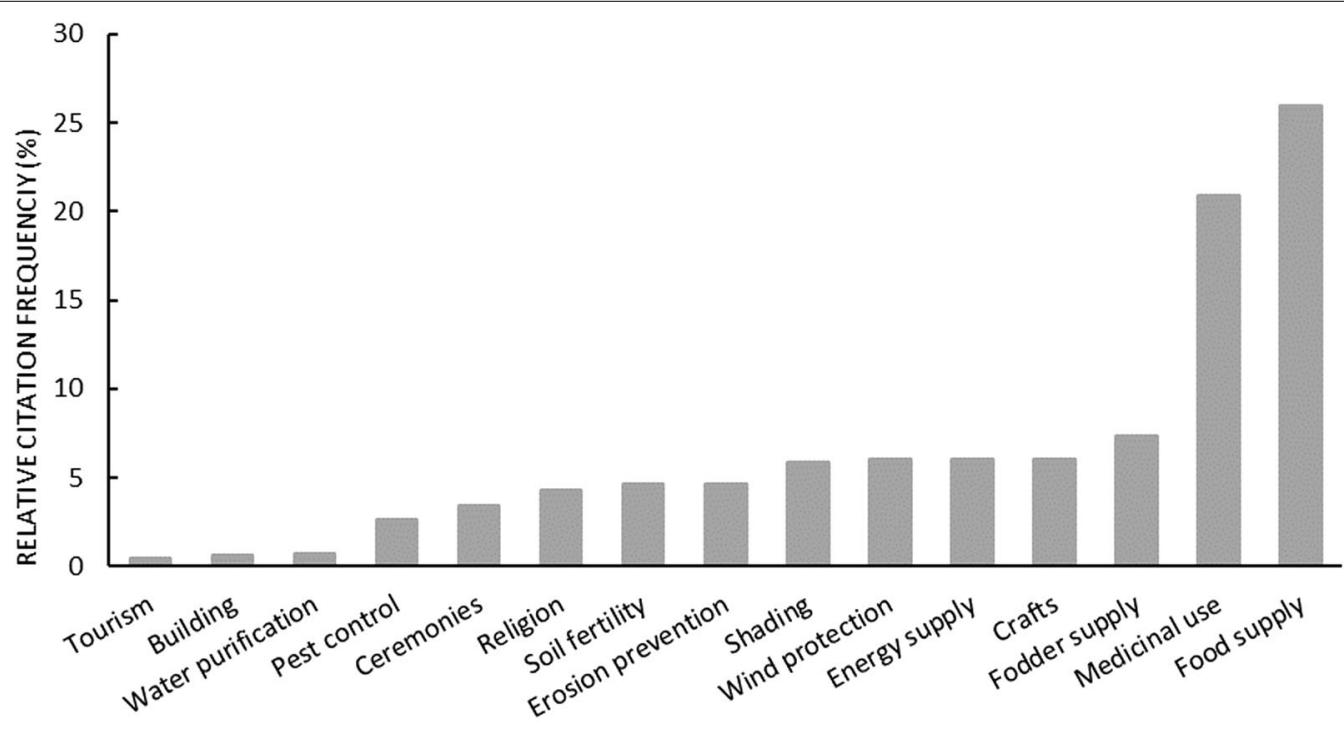

ECOSYSTEM SERVICES

Fig. 3 Relative frequency of citation of ecosystem services provided by plant species

\section{Variables influencing local knowledge of ecosystem} services

With regard to the knowledge of local populations in different land-use intensity areas (Table 4 ), the citation of ES varied significantly $(p<0.05)$, except for soil fertility, water purification and pest control services $(p>0.05)$. With regard to the sociocultural groups (Table 4$)$, the citation of ES varied significantly $(p<0.05)$ except for pest control services $(p>0.05)$. With regard to the gender of the informant, the citation of ES varied significantly 


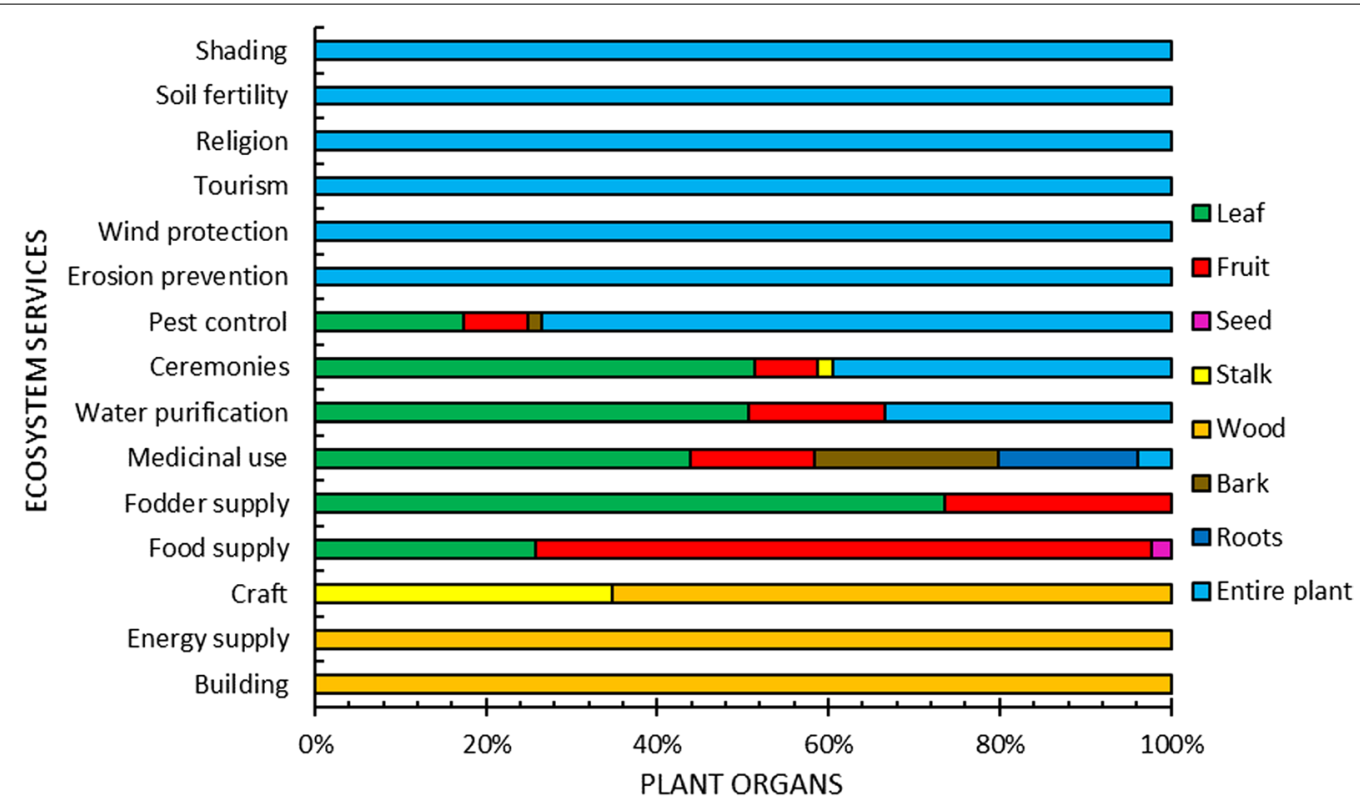

Fig. 4 Percentage of plant organs used for ecosystem services

$(p<0.05)$ only for food supply, fodder supply, crafts and soil fertility services. With regard to age classes, the citation of ES varied significantly $(p<0.05)$ only for medicinal use. The two variables associated with most differentiated citation of ES are the land use intensity levels and the sociocultural groups.

In order to understand a combined effect of land use area and sociocultural group, six sociocultural groups at the three land use sites were subjected to a cluster analysis, based on the citations of utilized ES (Fig. 5). These are: the groups of GRN Kassena, GRN Mossi, TWRB Mossi, CAD Pougouli, CAD Dagara and TWRB Dagara. The cluster analysis discriminated three groups, one comprising the Kassena and the Mossi at GRN, which is very different from the other two being composed of the natives at TWRB and CAD (the Dagara and the Pougouli) on the one hand and the Mossi at TWRB on the other hand.

Using the same data matrix, a principal component analysis grouped the $15 \mathrm{ES}$ (initial variables) into 5 synthetic variables or principal components. The two first principal components are represented by the graph of the PCA ordination (Fig. 6). The first component (Axis 1) explains $88.08 \%$ of the total variation, the second component (axis 2) 8.04\%. Therefore, these two axes explaining $96.12 \%$ of the total variation were used to describe relationships between sociocultural groups at different sites and ES. Axis 1 discriminated the natives of CAD and TWRB from the immigrant Mossi and the Kassena native to GRN. According to this axis, the Mossi of TWRB make use of the 15 ES only to a small degree and GRN residents mainly use the craft and tourism service while the natives of CAD and TWRB utilize the majority of services. Axis 2 discriminates ES utilization by the Mossi of TWRB from that by the GRN residents. Axis 2 underlines that the Mossi of TWRB make use of the 15 ES only to a small degree. The principal component analysis indicates that the utilization of ES is a function of the level of land use intensity and the economic benefits that people derive from plant formations. Thus, the GRN populations utilize the craft services related to tourism as they benefit from the financial income from tourism and participate in the management of the ranch. However, the natives of TWRB who do not benefit financially from the wildlife reserve of Bontioli and the natives of the anthropogenically shaped CAD utilize the ES that sustain the quality of the agricultural land and meet the primary needs of the rural populations.

\section{Vulnerability of the species}

The vulnerability indices of the used species in the study area varied from 1.57 to 2.71 (Table 3), indicating varying utilization pressures by local populations. Adansonia digitata, Afzelia africana, Diospyros mespiliformis, Khaya senegalensis, Lannea microcarpa and Pterocarpus erinaceus were most vulnerable with IV $=2.71$. Eighteen other species were highly vulnerable with IV $>2.5$ (Table 3 ). The ten most utilized species were highly vulnerable, except for Faidherbia albida and Vitellaria paradoxa classified as moderately vulnerable $\left(2^{<} \mathrm{IV} \leq 2.5\right)$. 
Table 3 Plant taxa utilized in the study area, their use values, and vulnerability indexes

\begin{tabular}{|c|c|c|c|c|c|c|c|}
\hline Plant taxon & Plant family & $\begin{array}{l}\text { Number of } \\
\text { reported } \\
\text { uses }\end{array}$ & $\begin{array}{l}\text { Number } \\
\text { of utilized } \\
\text { services }\end{array}$ & Used plant parts & Use Value & $\begin{array}{l}\text { Vulnerability } \\
\text { index }\end{array}$ & Voucher number \\
\hline Acacia spp. (ex) & Fabaceae & 9 & 5 & Lf. Wd. Ent & 0.04 & 2.43 & \\
\hline Adansonia digitata $\mathrm{L}$ & Malvaceae & 447 & 12 & Lf. Fr. Se. Wd. Bk. Ent & 1.693 & 2.71 & Ouédraogo. J. 61 (OUA) \\
\hline $\begin{array}{l}\text { Afzelia africana Sm. } \\
\text { ex Pers }\end{array}$ & Fabaceae & 343 & 12 & $\begin{array}{l}\text { Lf. Fr. Wd. St. Bk. Rt. } \\
\text { Ent }\end{array}$ & 1.337 & 2.71 & Guinko 1603 (OUA) \\
\hline $\begin{array}{l}\text { Agave sisalana Per- } \\
\text { rine [cult.] }\end{array}$ & Agavaceae & 11 & 3 & Ent & 0.05 & 2.29 & Yacouba H. 5399 (OUA) \\
\hline $\begin{array}{l}\text { Anacardium occiden- } \\
\text { tale L. [cult.] }\end{array}$ & Anacardiaceae & 29 & 8 & $\begin{array}{l}\text { Lf. Fr. Se. Wd. St. Bk. } \\
\text { Ent }\end{array}$ & 0.12 & 2.29 & $\begin{array}{l}\text { Thiombiano \& al. } 3249 \\
\text { (FR; OUA) }\end{array}$ \\
\hline $\begin{array}{l}\text { Andropogon chinensis } \\
\text { (Nees) Merr }\end{array}$ & Poaceae & 60 & 6 & Lf. St. Ent & 0.02 & 2.14 & Thiombiano 319 (OUA) \\
\hline $\begin{array}{l}\text { Andropogon gayanus } \\
\text { Kunth }\end{array}$ & Poaceae & 206 & 8 & Lf. St. Ent & 0.723 & 2.29 & Thiombiano 429 (OUA) \\
\hline Andropogon spp. & Poaceae & 18 & 3 & Lf. St. Ent & 0.08 & 2 & \\
\hline $\begin{array}{l}\text { Annona senegalensis } \\
\text { Pers }\end{array}$ & Anacardiaceae & 74 & 7 & $\begin{array}{l}\text { Lf. Fr. Fl. Wd. Bk. Rt. } \\
\text { Ent }\end{array}$ & 0.303 & 2.57 & Ouédraogo. J. 9 (OUA) \\
\hline $\begin{array}{l}\text { Azadirachta indica } \\
\text { A.Juss. [cult.] }\end{array}$ & Meliaceae & 307 & 11 & $\begin{array}{l}\text { Lf. Fr. Se. Fl. Wd. Bk. } \\
\text { Rt. Ent }\end{array}$ & 1.127 & 2.43 & Kristensen 26 (OUA) \\
\hline $\begin{array}{l}\text { Balanites aegyptiaca } \\
\text { (L.) Delile }\end{array}$ & Zygophyllaceae & 175 & 9 & Lf. Fr. Wd. Bk. Rt. Ent & 0.497 & 2.57 & Ouédraogo. J. 4 (OUA) \\
\hline Boerhavia diffusa $\mathrm{L}$ & Nyctaginaceae & 9 & 1 & Ent & 0.03 & 1.86 & $\begin{array}{l}\text { Thiombiano \& al. } 95 \\
\text { (OUA) }\end{array}$ \\
\hline $\begin{array}{l}\text { Bombax costatum } \\
\text { Pellegr. \& Vuill }\end{array}$ & Malvaceae & 97 & 9 & $\begin{array}{l}\text { Lf. Fr. Fl. Wd. Bk. Rt. } \\
\text { Ent }\end{array}$ & 0.39 & 2.57 & $\begin{array}{l}\text { Tiné \& Bambara } 29 \\
\text { (OUA) }\end{array}$ \\
\hline $\begin{array}{l}\text { Bridelia scleroneura } \\
\text { Müll. Arg }\end{array}$ & Phyllanthaceae & 10 & 5 & Wd. Bk. Rt. Ent & 0.047 & 2.43 & $\begin{array}{l}\text { Guinko \& al. } 3388 \\
\text { (OUA) }\end{array}$ \\
\hline Burkea africana Hook & Fabaceae & 41 & 7 & Lf. Wd. Bk. Rt. Ent & 0.19 & 2.43 & Mbayngone 335 (OUA) \\
\hline $\begin{array}{l}\text { Cadaba farinosa } \\
\text { Forssk }\end{array}$ & Capparaceae & 5 & 4 & Lf. Rt. Ent & 0.017 & 2.29 & $\begin{array}{l}\text { Ouédraogo. A. } 86 \\
\text { (OUA) }\end{array}$ \\
\hline $\begin{array}{l}\text { Calotropis procera } \\
\text { (Ait.) Ait. f }\end{array}$ & Apocynaceae & 8 & 3 & Lf. Fr. Rt. Ent & 0.033 & 2.29 & $\begin{array}{l}\text { Guinko \& al. } 3423 \\
\text { (OUA) }\end{array}$ \\
\hline Capparis sepiaria $\mathrm{L}$ & Capparaceae & 11 & 7 & Lf. Fr. Rt. Ent & 0.04 & 2.43 & $\begin{array}{l}\text { Tiné \& Bambara } 53 \\
\text { (OUA) }\end{array}$ \\
\hline $\begin{array}{l}\text { Capsicum frutescens } \\
\text { L. [cult.] }\end{array}$ & Solanaceae & 9 & 1 & Lf. Fr. Ent & 0.037 & 2.14 & $\begin{array}{l}\text { Thiombianoet al. } 3172 \\
\text { (OUA) }\end{array}$ \\
\hline $\begin{array}{l}\text { Carica papaya L. } \\
\text { [cult.] }\end{array}$ & Caricaceae & 76 & 3 & Lf. Fr & 0.283 & 2 & N'Do 6806 (OUA) \\
\hline Cassia nigricans Vahl & Fabaceae & 62 & 4 & Lf. Fr. Ent & 0.283 & 2.14 & $\begin{array}{l}\text { Thiombiano \& al. } 2248 \\
\text { (OUA) }\end{array}$ \\
\hline Cassia obtusifolia L & Fabaceae & 13 & 1 & Lf & 0.057 & 1.57 & Guinko 62 (OUA) \\
\hline Cassia sieberiana DC & Fabaceae & 33 & 4 & Lf. Fr. Wd. Bk. Rt. Ent & 0.107 & 2.29 & Guinko 1340 (OUA \\
\hline $\begin{array}{l}\text { Ceiba pentandra (L.) } \\
\text { Gaertn }\end{array}$ & Fabaceae & 2 & 2 & Ent & 0.007 & 2.29 & Kristensen 47 (OUA) \\
\hline $\begin{array}{l}\text { Chasmopodium cau- } \\
\text { datum (Hack.) Stapf }\end{array}$ & Malvaceae & 64 & 11 & Lf. Fr. Se. Wd. Bk. Ent & 0.21 & 2.43 & Tibiri A. 4390 (OUA) \\
\hline $\begin{array}{l}\text { Chrysopogon } \\
\text { nigritanus (Benth.) } \\
\text { Veldkamp }\end{array}$ & Poaceae & 19 & 2 & Ent & 0.073 & 2 & Mbayngone 133 (OUA) \\
\hline $\begin{array}{l}\text { Citrus aurantium L. } \\
\text { [cult.] }\end{array}$ & Poaceae & 3 & 1 & Lf. Ent & 0.01 & 1.86 & Guinko 1023 (OUA) \\
\hline $\begin{array}{l}\text { Citrus limon (L.) } \\
\text { Burm.f. [cult.] }\end{array}$ & Rutaceae & 24 & 4 & Lf. Fr. Se. Wd. Ent & 0.107 & 2.14 & \\
\hline $\begin{array}{l}\text { Cochlospermum } \\
\text { planchonii Hook. f. ex } \\
\text { Planch }\end{array}$ & Rutaceae & 107 & 4 & Lf. Fr. Se.Wd. Ent & 0.387 & 2.14 & Küppers 1293 (FR) \\
\hline
\end{tabular}


Table 3 (continued)

\begin{tabular}{|c|c|c|c|c|c|c|c|}
\hline Plant taxon & Plant family & $\begin{array}{l}\text { Number of } \\
\text { reported } \\
\text { uses }\end{array}$ & $\begin{array}{l}\text { Number } \\
\text { of utilized } \\
\text { services }\end{array}$ & Used plant parts & Use Value & $\begin{array}{l}\text { Vulnerability } \\
\text { index }\end{array}$ & Voucher number \\
\hline $\begin{array}{l}\text { Cola cordifolia (Cav.) } \\
\text { R.Br }\end{array}$ & Bixaceae & 3 & 2 & Fr. Se. Rt. Ent & 0.013 & 2 & Guinko 57 (OUA) \\
\hline Cola laurifolia Mast & Malvaceae & 7 & 1 & $\mathrm{Fr}$ & 0.033 & 2 & $\begin{array}{l}\text { Schmidt \& al. } 875 \\
\text { (OUA) }\end{array}$ \\
\hline $\begin{array}{l}\text { Coldenia procum- } \\
\text { bens } L\end{array}$ & Malvaceae & 4 & 2 & $\mathrm{Fr}$ & 0.02 & 2.14 & Thiombiano 210 (OUA) \\
\hline $\begin{array}{l}\text { Combretum adenogo- } \\
\text { nium Steud. ex A. } \\
\text { Rich }\end{array}$ & Boraginaceae & 3 & 2 & Lf & 0.013 & 1.86 & Guinko 612 (OUA) \\
\hline $\begin{array}{l}\text { Combretum collinum } \\
\text { Fresen }\end{array}$ & Combretaceae & 24 & 7 & Lf. Fr. Wd. Rt. Ent & 0.13 & 2.43 & Thiombiano 729 (OUA) \\
\hline $\begin{array}{l}\text { Combretum glutino- } \\
\text { sum Perr. ex DC }\end{array}$ & Combretaceae & 65 & 7 & Lf. Fr. Wd. Bk. Rt. Ent & 0.243 & 2.43 & Guinko 1069 (OUA) \\
\hline $\begin{array}{l}\text { Combretum molle } \\
\text { G. Don }\end{array}$ & Combretaceae & 1 & 1 & Wd & 0.003 & 2.14 & $\begin{array}{l}\text { Thiombiano \& al. } 2442 \\
\text { (OUA) }\end{array}$ \\
\hline $\begin{array}{l}\text { Combretum nigri- } \\
\text { cans Lepr. ex Guill. } \\
\& \text { Perr }\end{array}$ & Combretaceae & 3 & 1 & Lf & 0.013 & 1.86 & Thiombiano 84 (OUA) \\
\hline $\begin{array}{l}\text { Combretum panicula- } \\
\text { tum Vent }\end{array}$ & Combretaceae & 1 & 1 & Wd & 0.003 & 1.86 & $\begin{array}{l}\text { Thiombiano \& al. } 2465 \\
\text { (OUA) }\end{array}$ \\
\hline $\begin{array}{l}\text { Combretum sericeum } \\
\text { G. Don }\end{array}$ & Combretaceae & 10 & 2 & Lf. Rt. Ent & 0.03 & 2.29 & $\begin{array}{l}\text { Thiombiano \& al. } 2003 \\
\text { (OUA) }\end{array}$ \\
\hline Corchorus olitorius L & Combretaceae & 7 & 2 & Lf. Fr & 0.023 & 2.14 & Thiombiano 607 (OUA) \\
\hline Cordia myxa L & Malvaceae & 18 & 6 & Lf. Fr. Ent & 0.08 & 2.14 & Ouoba 39 (OUA) \\
\hline Crateva adansonii DC & Boraginaceae & 180 & 11 & Lf. Fr. Wd. St. Bk. Ent & 0.75 & 2.57 & Ouoba 36 (OUA) \\
\hline $\begin{array}{l}\text { Crossopteryx febrifuga } \\
\text { (Afzel. ex G.Don) } \\
\text { Benth }\end{array}$ & Capparaceae & 131 & 8 & Lf. Fr. Wd. Bk. Rt. Ent & 0.537 & 2.57 & $\begin{array}{l}\text { Tiné \& Bambara } 52 \\
\text { (OUA) }\end{array}$ \\
\hline Cyanotis lanata Benth & Rubiaceae & 65 & 7 & Lf. Fr. Wd. Bk. Rt. Ent & 0.23 & 2.43 & Madsen 5159 (OUA) \\
\hline $\begin{array}{l}\text { Cymbopogon caesius } \\
\text { (Nees ex Hook. \& } \\
\text { Arn.) Stapf }\end{array}$ & Commelinaceae & 5 & 1 & Ent & 0.023 & 1.86 & $\begin{array}{l}\text { Thiombiano } 1029 \\
\text { (OUA) }\end{array}$ \\
\hline $\begin{array}{l}\text { Cymbopogon schoe- } \\
\text { nanthus (L.) Spreng }\end{array}$ & Poaceae & 34 & 7 & Lf. St. Ent & 0.113 & 2.14 & $\begin{array}{l}\text { Laegaard \& al. } 18306 \\
\text { (OUA) }\end{array}$ \\
\hline $\begin{array}{l}\text { Dalbergia boehmii } \\
\text { Taub }\end{array}$ & Poaceae & 31 & 7 & Lf. Rt. Ent & 0.143 & 2.14 & Korbéogo 12 (OUA) \\
\hline $\begin{array}{l}\text { Dalbergia melanoxy- } \\
\text { Ion Guill. \& Perr }\end{array}$ & Fabaceae & 1 & 1 & $W d$ & 0.003 & 2.14 & Ouattara 75 (OUA) \\
\hline $\begin{array}{l}\text { Daniellia oliveri (Rolfe) } \\
\text { Hutch. \& Dalziel }\end{array}$ & Fabaceae & 94 & 10 & Lf. Wd. St. Bk. Rt. Ent & 0.36 & 2.57 & $\begin{array}{l}\text { Ouédraogo. A. } 17 \\
\text { (OUA) }\end{array}$ \\
\hline $\begin{array}{l}\text { Detarium microcar- } \\
\text { pum Guill. \& Perr }\end{array}$ & Fabaceae & 338 & 13 & $\begin{array}{l}\text { Lf. Fr. Se. Wd. Bk. Rt. } \\
\text { Ent }\end{array}$ & 1.21 & 2.57 & Thiombiano 808 (OUA) \\
\hline $\begin{array}{l}\text { Dichrostachys cinerea } \\
\text { (L.) Wight \& Arn }\end{array}$ & Fabaceae & 14 & 7 & Lf. Wd. Ent & 0.047 & 2.43 & Ouoba 2001 (OUA) \\
\hline $\begin{array}{l}\text { Dicoma tomentosa } \\
\text { Cass }\end{array}$ & Asteraceae & 1 & 1 & Ent & 0.003 & 1.86 & Madsen 5926 (OUA) \\
\hline $\begin{array}{l}\text { Diospyros mespili- } \\
\text { formis Hochst. ex } \\
\text { A. DC }\end{array}$ & Ebenaceae & 402 & 14 & $\begin{array}{l}\text { Lf. Fr. Se. Wd. St. Bk. } \\
\text { Rt. Ent }\end{array}$ & 2.249 & 2.71 & Ouédraogo. J. 35 (OUA) \\
\hline $\begin{array}{l}\text { Echinochloa pyrami- } \\
\text { dalis (Lam.) Hitchc. \& } \\
\text { Chase }\end{array}$ & Poaceae & 23 & 3 & St. Ent & 0.077 & 2 & Laegaard 21295 (OUA) \\
\hline $\begin{array}{l}\text { Entada africana Guill. } \\
\text { \& Perr }\end{array}$ & Fabaceae & 32 & 5 & Wd. Bk. Ent & 0.15 & 2.43 & Guinko 733 (OUA) \\
\hline
\end{tabular}


Table 3 (continued)

\begin{tabular}{|c|c|c|c|c|c|c|c|}
\hline Plant taxon & Plant family & $\begin{array}{l}\text { Number of } \\
\text { reported } \\
\text { uses }\end{array}$ & $\begin{array}{l}\text { Number } \\
\text { of utilized } \\
\text { services }\end{array}$ & Used plant parts & Use Value & $\begin{array}{l}\text { Vulnerability } \\
\text { index }\end{array}$ & Voucher number \\
\hline $\begin{array}{l}\text { Erythrina senegalensis } \\
\text { A.DC }\end{array}$ & Fabaceae & 4 & 3 & Lf. Bk. Ent & 0.02 & 2.29 & Guinko 116 (OUA) \\
\hline $\begin{array}{l}\text { Eucalyptus camaldu- } \\
\text { lensis Dehnh. [cult.] }\end{array}$ & Myrtaceae & 78 & 9 & Lf. Fr. Wd. Bk. Rt. Ent & 0.32 & 2.29 & Rosendal 6876 (OUA) \\
\hline $\begin{array}{l}\text { Fadogia agrestis } \\
\text { Schweinf. ex Hiern }\end{array}$ & Rubiaceae & 2 & 2 & Lf. Ent & 0.007 & 2.29 & $\begin{array}{l}\text { Thiombiano \& al. } 21 \\
\text { (OUA) }\end{array}$ \\
\hline $\begin{array}{l}\text { Faidherbia albida } \\
\text { (Delile) A. Chev. Del }\end{array}$ & Fabaceae & 384 & 11 & Lf. Fr. Wd. Bk. Rt. Ent & 1.53 & 2.43 & Tibiri A. 4369 (OUA) \\
\hline $\begin{array}{l}\text { Feretia apodanthera } \\
\text { Delile }\end{array}$ & Rubiaceae & 20 & 5 & Lf. Wd. Bk. Rt. Ent & 0.083 & 2.43 & Guinko 1370 (OUA) \\
\hline $\begin{array}{l}\text { Ficus abutilifolia (Miq.) } \\
\text { Miq }\end{array}$ & Moraceae & 12 & 4 & Lf. Fr.Wd. Ent & 0.037 & 2.29 & Thiombiano 238 (OUA) \\
\hline $\begin{array}{l}\text { Ficus dicranostyla } \\
\text { Mildbr }\end{array}$ & Moraceae & 9 & 2 & Lf. Fr. Bk & 0.037 & 2.29 & $\begin{array}{l}\text { Schmidt \& al. } 913 \\
\text { (OUA) }\end{array}$ \\
\hline $\begin{array}{l}\text { Ficus ingens (Miq.) } \\
\text { Miq }\end{array}$ & Moraceae & 1 & 1 & $\mathrm{Fr}$ & 0.033 & 2 & $\begin{array}{l}\text { Ouédraogo. O. } 104 \\
\text { (OUA) }\end{array}$ \\
\hline $\begin{array}{l}\text { Ficus platyphylla } \\
\text { Delile }\end{array}$ & Moraceae & 22 & 7 & Lf. Fr. Wd. Bk. Ent & 0.083 & 2.43 & $\begin{array}{l}\text { Guinko \& al. } 6004 \\
\text { (OUA) }\end{array}$ \\
\hline Ficus sur Forssk & Moraceae & 6 & 3 & Fr. Bk. Ent & 0.02 & 2.29 & Ouoba 19 (OUA) \\
\hline Ficus sycomorus $\mathrm{L}$ & Moraceae & 337 & 12 & $\begin{array}{l}\text { Lf. Fr. Wd. St. Bk. Rt. } \\
\text { Ent }\end{array}$ & 1.273 & 2.57 & $\begin{array}{l}\text { Ouédraogo. A. } 133 \\
\text { (OUA) }\end{array}$ \\
\hline $\begin{array}{l}\text { Flacourtia indica } \\
\text { (Burm. f.) Merrill }\end{array}$ & Salicaceae & 4 & 2 & Lf. Fr & 0.02 & 2.14 & Ouoba 18 (OUA) \\
\hline $\begin{array}{l}\text { Flueggea virosa (Roxb. } \\
\text { ex Willd.) Voigt }\end{array}$ & Phyllanthaceae & 6 & 2 & Lf. Wd. St & 0.02 & 2.29 & Thiombiano 226 (OUA) \\
\hline $\begin{array}{l}\text { Gardenia erubescens } \\
\text { Stapf \& Hutch }\end{array}$ & Rubiaceae & 196 & 10 & Lf. Fr. Wd. Bk. Rt. Ent & 0.857 & 2.57 & Guinko 374 (OUA) \\
\hline $\begin{array}{l}\text { Gardenia ternifolia } \\
\text { Schumach. \& Thonn }\end{array}$ & Rubiaceae & 3 & 2 & Fr. Rt. Ent & 0.013 & 2.29 & $\begin{array}{l}\text { Thiombiano \& al. } 285 \\
\text { (OUA) }\end{array}$ \\
\hline $\begin{array}{l}\text { Gmelina arborea } \\
\text { Roxb. [cult.] }\end{array}$ & Lamiaceae & 16 & 6 & Lf. Fr. Fl. Ent & 0.07 & 2.43 & $\begin{array}{l}\text { Thiombiano \& al. } 43 \\
\text { (OUA) }\end{array}$ \\
\hline Grewia bicolor Juss & Malvaceae & 2 & 1 & $\mathrm{Fr}$ & 0.007 & 2 & $\begin{array}{l}\text { Thiombiano \& al. } 407 \\
\text { (OUA) }\end{array}$ \\
\hline $\begin{array}{l}\text { Grewia cissoides } \\
\text { Hutch. \& Dalziel }\end{array}$ & Malvaceae & 1 & 1 & $\mathrm{Fr}$ & 0.003 & 2 & $\begin{array}{l}\text { Thiombiano \& al. } 191 \\
\text { (OUA) }\end{array}$ \\
\hline $\begin{array}{l}\text { Grewia lasiodiscus } \\
\text { K.Schum }\end{array}$ & Malvaceae & 8 & 2 & Lf. Fr. Wd & 0.037 & 2.29 & Thiombiano 614 (OUA) \\
\hline Grewia spp & Malvaceae & 3 & 2 & Ent & 0.01 & 2.29 & \\
\hline $\begin{array}{l}\text { Guiera senegalensis } \\
\text { J.F.Gmel }\end{array}$ & Combretaceae & 24 & 2 & Lf. St. Ent & 0.08 & 2.29 & $\begin{array}{l}\text { Ouédraogo. A. } 239 \\
\text { (OUA) }\end{array}$ \\
\hline $\begin{array}{l}\text { Gymnosporia senega- } \\
\text { lensis (Lam.) Loes }\end{array}$ & Celastraceae & 21 & 6 & Lf. Fl. Wd. Bk. Rt. Ent & 0.083 & 2.29 & $\begin{array}{l}\text { Schmidt \& al. } 912 \\
\text { (OUA) }\end{array}$ \\
\hline $\begin{array}{l}\text { Haematostaphis } \\
\text { barteri Hook.f }\end{array}$ & Anacardiaceae & 4 & 1 & Lf & 0.013 & 1.86 & $\begin{array}{l}\text { Ouédraogo. A. } 102 \\
\text { (OUA) }\end{array}$ \\
\hline $\begin{array}{l}\text { Hibiscus cannabinus } \\
\text { L. [cult.] }\end{array}$ & Malvaceae & 12 & 3 & Lf. Fr. Se. Fl. Ent & 0.037 & 2 & $\begin{array}{l}\text { Ouédraogo. O. } 364 \\
\text { (OUA) }\end{array}$ \\
\hline $\begin{array}{l}\text { Hymenocardia acida } \\
\text { Tul }\end{array}$ & Phyllanthaceae & 9 & 2 & Lf. Wd & 0.03 & 2.29 & Guinko 888 (OUA) \\
\hline Hyparrhenia spp & Poaceae & 2 & 2 & Ent & 0.007 & 2 & $\begin{array}{l}\text { Ouoba } 157(\text { OUA); } \\
\text { Madsen } 6003(\text { OUA); } \\
\text { Mbayngone } 125 \text { (OUA) }\end{array}$ \\
\hline Hyptis spicigera Lam & Lamiaceae & 86 & 6 & Lf. Ent & 0.37 & 2.29 & Guinko 610 (OUA) \\
\hline $\begin{array}{l}\text { Indigofera bracteolata } \\
\text { DC }\end{array}$ & Fabaceae & 2 & 2 & Rt. Ent & 0.01 & 2 & Ouattara 71 (OUA) \\
\hline Ipomoea carnea Jacq & Convolvulaceae & 3 & 1 & Lf. Ent & 0.007 & 2 & Guinko 1290 (OUA) \\
\hline
\end{tabular}


Table 3 (continued)

\begin{tabular}{|c|c|c|c|c|c|c|c|}
\hline Plant taxon & Plant family & $\begin{array}{l}\text { Number of } \\
\text { reported } \\
\text { uses }\end{array}$ & $\begin{array}{l}\text { Number } \\
\text { of utilized } \\
\text { services }\end{array}$ & Used plant parts & Use Value & $\begin{array}{l}\text { Vulnerability } \\
\text { index }\end{array}$ & Voucher number \\
\hline $\begin{array}{l}\text { Isoberlinia doka Craib } \\
\text { \& Stapf }\end{array}$ & Fabaceae & 69 & 10 & Lf. Fr. Wd. Bk. Ent & 0.26 & 2.57 & $\begin{array}{l}\text { Thiombiano \& al. } 319 \\
\text { (OUA); }\end{array}$ \\
\hline $\begin{array}{l}\text { Jatropha curcas L. } \\
\text { [cult.] }\end{array}$ & Euphorbiaceae & 31 & 8 & Ent & 0.123 & 2.29 & Tibiri A. 4403 (OUA) \\
\hline $\begin{array}{l}\text { Khaya senegalensis } \\
\text { (Desv.) A. Juss }\end{array}$ & Meliaceae & 403 & 14 & Lf. Fr. Wd. Bk. Rt. Ent & 1.503 & 2.71 & Sawadogo 6883 (OUA) \\
\hline $\begin{array}{l}\text { Kigelia africana (Lam.) } \\
\text { Benth }\end{array}$ & Bignoniaceae & 19 & 4 & Lf. Fr. Bk. Rt. Ent & 0.077 & 2.29 & Thiombiano 469 (OUA) \\
\hline $\begin{array}{l}\text { Landolphia heudelotii } \\
\text { A. DC }\end{array}$ & Apocynaceae & 13 & 3 & Lf. Fr. St & 0.05 & 2.29 & $\begin{array}{l}\text { Guinko \& al. } 5085 \\
\text { (OUA) }\end{array}$ \\
\hline Lannea acida A. Rich & Anacardiaceae & 55 & 10 & $\begin{array}{l}\text { Lf. Fr. Wd. St. Bk. Rt. } \\
\text { Ent }\end{array}$ & 0.207 & 2.29 & Ouédraogo. J. 87 (OUA) \\
\hline $\begin{array}{l}\text { Lannea microcarpa } \\
\text { Engl. \& K. Krause }\end{array}$ & Anacardiaceae & 371 & 13 & Lf. Fr. Wd. Bk. Rt. Ent & 1.583 & 2.71 & Guinko 1383 (OUA) \\
\hline $\begin{array}{l}\text { Lannea velutina } \\
\text { A.Rich }\end{array}$ & Anacardiaceae & 1 & 1 & Bk & 0.003 & 2.14 & $\begin{array}{l}\text { Ouédraogo. A. } 105 \\
\text { (OUA) }\end{array}$ \\
\hline $\begin{array}{l}\text { Leptadenia hastata } \\
\text { (Pers.) Decne }\end{array}$ & Apocynaceae & 14 & 4 & Lf. Fr. Fl. Rt. Ent & 0.06 & 2.29 & $\begin{array}{l}\text { Thiombiano \& al. } 2028 \\
\text { (OUA) }\end{array}$ \\
\hline $\begin{array}{l}\text { Loeseneriella africana } \\
\text { (Willd.) N.Hallé }\end{array}$ & Celastraceae & 1 & 1 & Lf & 0.003 & 1.86 & $\begin{array}{l}\text { Ouédraogo. O. } 67 \\
\text { (OUA) }\end{array}$ \\
\hline $\begin{array}{l}\text { Lophira lanceolata } \\
\text { Tiegh. ex Keay }\end{array}$ & Ochnaceae & 78 & 5 & Lf. Fr. Wd. Bk. Rt. Ent & 0.337 & 2.43 & Guinko 2063 (OUA) \\
\hline $\begin{array}{l}\text { Loudetia simplex } \\
\text { (Nees) C.E.Hubb }\end{array}$ & Poaceae & 2 & 2 & Lf. St & 0.01 & 1.86 & $\begin{array}{l}\text { Laegaard \& al. } 21140 \\
\text { (OUA) }\end{array}$ \\
\hline $\begin{array}{l}\text { Loudetia togoensis } \\
\text { (Pilg.) C.E.Hubb }\end{array}$ & Poaceae & 1 & 1 & Ent & 0.003 & 1.86 & Mbayngone 45 (OUA) \\
\hline $\begin{array}{l}\text { Maerua angolensis } \\
\text { DC }\end{array}$ & Capparaceae & 7 & 2 & Lf. Fl. Wd & 0.023 & 2.43 & $\begin{array}{l}\text { Ouédraogo. A. } 20 \\
\text { (OUA) }\end{array}$ \\
\hline $\begin{array}{l}\text { Maerua crassifolia } \\
\text { Forssk }\end{array}$ & Capparaceae & 3 & 2 & Lf. Ent & 0.013 & 2.29 & Guinko 2350 (OUA) \\
\hline $\begin{array}{l}\text { Mangifera indica } \mathrm{L} \text {. } \\
\text { [cult.] }\end{array}$ & Anacardiaceae & 359 & 13 & Lf. Fr. Wd. Bk. Rt. Ent & 1.617 & 2.43 & $\begin{array}{l}\text { Ouédraogo. H. } 35 \\
\text { (OUA) }\end{array}$ \\
\hline Mimosa pigra L & Fabaceae & 5 & 2 & Wd. Ent & 0.017 & 2.29 & Guinko 605 (OUA) \\
\hline $\begin{array}{l}\text { Mitragyna inermis } \\
\text { (Willd.) Kuntze }\end{array}$ & Rubiaceae & 53 & 9 & $\begin{array}{l}\text { Lf. Fr. Wd. St. Bk. Rt. } \\
\text { Ent }\end{array}$ & 0.433 & 2.43 & $\begin{array}{l}\text { Schmidt \& al. } 6369 \\
\text { (OUA) }\end{array}$ \\
\hline Moringa oleifera $\mathrm{L}$ & Moringaceae & 157 & 8 & Lf. Fr. Wd. Bk. Rt. Ent & 0.6 & 2.43 & $\begin{array}{l}\text { Thiombiano \& al. } 3877 \\
\text { (OUA) }\end{array}$ \\
\hline Nymphaea lotus $\mathrm{L}$ & Nymphaeaceae & 8 & 4 & Lf. Rt. Ent & 0.033 & 2.29 & Madsen 5649 (OUA) \\
\hline $\begin{array}{l}\text { Ocimum america- } \\
\text { num } \mathrm{L}\end{array}$ & Lamiaceae & 48 & 5 & Lf. Ent & 0.197 & 2.14 & $\begin{array}{l}\text { Guinko \& al. } 3489 \\
\text { (OUA) }\end{array}$ \\
\hline $\begin{array}{l}\text { Oncoba spinosa } \\
\text { Forssk }\end{array}$ & Salicaceae & 32 & 4 & Lf. Fr. Wd. Bk. Rt. Ent & 0.133 & 2.29 & $\begin{array}{l}\text { Thiombiano \& Boussim } \\
283 \text { (OUA); }\end{array}$ \\
\hline $\begin{array}{l}\text { Opilia amentacea } \\
\text { Roxb }\end{array}$ & Opiliaceae & 20 & 3 & Lf. Fr. Rt. Ent & 0.067 & 2.29 & Ouédraogo. J. 82 (OUA) \\
\hline $\begin{array}{l}\text { Oxytenanthera abys- } \\
\text { sinica (A.Rich.) Munro }\end{array}$ & Poaceae & 1 & 1 & St & 0.003 & 2 & $\begin{array}{l}\text { Schmidt \& al. } 893 \\
\text { (OUA) }\end{array}$ \\
\hline $\begin{array}{l}\text { Ozoroa obovata (Oliv.) } \\
\text { R.Fern. \& A.Fern }\end{array}$ & Anacardiaceae & 1 & 1 & Lf & 0.003 & 1.86 & $\begin{array}{l}\text { Thiombiano \& al. } 2449 \\
\text { (OUA) }\end{array}$ \\
\hline $\begin{array}{l}\text { Parinari curatellifolia } \\
\text { Planch. ex Benth }\end{array}$ & Chrysobalanaceae & 7 & 3 & Lf. Fr. Bk. Ent & 0.027 & 2.29 & $\begin{array}{l}\text { Ouédraogo. A. } 170 \\
\text { (OUA) }\end{array}$ \\
\hline $\begin{array}{l}\text { Parkia biglobosa } \\
\text { (Jacq.) R. Br. ex G. } \\
\text { Donf }\end{array}$ & Fabaceae & 584 & 12 & $\begin{array}{l}\text { Lf. Fr. Se. Wd. Bk. Rt. } \\
\text { Ent }\end{array}$ & 2.353 & 2.57 & Madsen 5113 (OUA) \\
\hline Paullinia pinnata $\mathrm{L}$ & Sapindaceae & 8 & 1 & Lf. Rt & 0.023 & 2.14 & Guinko 604 (OUA) \\
\hline
\end{tabular}


Table 3 (continued)

\begin{tabular}{|c|c|c|c|c|c|c|c|}
\hline Plant taxon & Plant family & $\begin{array}{l}\text { Number of } \\
\text { reported } \\
\text { uses }\end{array}$ & $\begin{array}{l}\text { Number } \\
\text { of utilized } \\
\text { services }\end{array}$ & Used plant parts & Use Value & $\begin{array}{l}\text { Vulnerability } \\
\text { index }\end{array}$ & Voucher number \\
\hline $\begin{array}{l}\text { Pennisetum pedicel- } \\
\text { latum Trin }\end{array}$ & Poaceae & 8 & 5 & Lf. St. Ent & 0.037 & 2.14 & $\begin{array}{l}\text { Thiombiano } 1007 \\
\text { (OUA) }\end{array}$ \\
\hline Pennisetum spp & Poaceae & 29 & 3 & St. Ent & 0.107 & 2 & $\begin{array}{l}\text { Thiombiano } 1007 \\
\text { (OUA) Laegaard \& al. } \\
18412 \text { (OUA) }\end{array}$ \\
\hline $\begin{array}{l}\text { Pericopsis laxiflora } \\
\text { (Benth. ex Bak.) van } \\
\text { Meeuwen }\end{array}$ & Fabaceae & 23 & 6 & Lf. Wd. Rt. Ent & 0.103 & 2.43 & Thiombiano 853 (OUA) \\
\hline $\begin{array}{l}\text { Philenoptera laxiflora } \\
\text { (Guill. \& Perr.) Roberty }\end{array}$ & Fabaceae & 29 & 8 & Lf. Fr. Wd. Bk. Rt. Ent & 0.117 & 2.43 & Taïta 203 (OUA) \\
\hline $\begin{array}{l}\text { Piliostigma reticula- } \\
\text { tum (DC.) Hochst }\end{array}$ & Fabaceae & 30 & 8 & Lf. Fr. Wd. St. Bk. Ent & 0.113 & 2.43 & Thiombiano 604 (OUA) \\
\hline $\begin{array}{l}\text { Piliostigma thonningii } \\
\text { (Schum.) Milne- } \\
\text { Redhead }\end{array}$ & Fabaceae & 135 & 11 & $\begin{array}{l}\text { Lf. Fr. Wd. St. Bk. Rt. } \\
\text { Ent }\end{array}$ & 0.563 & 2.43 & $\begin{array}{l}\text { Thiombiano \& al. } 2200 \\
\text { (OUA) }\end{array}$ \\
\hline $\begin{array}{l}\text { Prosopis africana } \\
\text { (Guill. \& Perr.) Taub }\end{array}$ & Fabaceae & 31 & 7 & Lf. Fr. Wd. Bk. Rt. Ent & 0.13 & 2.43 & Kristensen 44 (OUA) \\
\hline $\begin{array}{l}\text { Pseudocedrela } \\
\text { kotschyi (Schweinf.) } \\
\text { Harms }\end{array}$ & Meliaceae & 36 & 9 & Lf. Wd. Bk. Ent & 0.13 & 2.43 & Thiombiano 194 (OUA) \\
\hline $\begin{array}{l}\text { Psidium guajava } \mathrm{L} \text {. } \\
\text { [cult.] }\end{array}$ & Myrtaceae & 43 & 4 & Lf. Fr. Wd. Bk. Rt. Ent & 0.193 & 2.14 & $\begin{array}{l}\text { Ouédraogo. H. } 40 \\
\text { (OUA) }\end{array}$ \\
\hline $\begin{array}{l}\text { Pterocarpus erinaceus } \\
\text { Poir }\end{array}$ & Fabaceae & 297 & 13 & Lf. Fr. Wd. Bk. Rt. Ent & 1.237 & 2.71 & Guinko 1030 (OUA) \\
\hline $\begin{array}{l}\text { Pterocarpus santali- } \\
\text { noides DC }\end{array}$ & Fabaceae & 2 & 2 & Se.Wd & 0.007 & 2.29 & $\begin{array}{l}\text { Ouédraogo. O. } 177 \\
\text { (OUA) }\end{array}$ \\
\hline $\begin{array}{l}\text { Raphionacme splend- } \\
\text { ens Schltr }\end{array}$ & Apocynaceae & 2 & 2 & Rt. Ent & 0.01 & 2.29 & $\begin{array}{l}\text { Thiombiano \& al. } 2833 \\
\text { (OUA) }\end{array}$ \\
\hline $\begin{array}{l}\text { Rottboellia cochin- } \\
\text { chinensis (Lour.) } \\
\text { Clayton }\end{array}$ & Poaceae & 85 & 8 & Lf. Ent & 0.4 & 2.29 & Guinko 1890 (OUA) \\
\hline $\begin{array}{l}\text { Rytigynia senegalensis } \\
\text { Blume }\end{array}$ & Rubiaceae & 1 & 1 & Wd & 0.003 & 2.14 & $\begin{array}{l}\text { Schmidt \& al. } 1189 \text { (FR; } \\
\text { OUA) }\end{array}$ \\
\hline $\begin{array}{l}\text { Saba senegalensis (A. } \\
\text { DC.) Pichon }\end{array}$ & Apocynaceae & 239 & 14 & $\begin{array}{l}\text { Lf. Fr. Se. Wd. St. Bk. } \\
\text { Rt. Ent }\end{array}$ & 1.027 & 2.57 & $\begin{array}{l}\text { Guinko \& al. } 6000 \\
\text { (OUA) }\end{array}$ \\
\hline $\begin{array}{l}\text { Sarcocephalus latifo- } \\
\text { lius (Sm.) E.A.Bruce }\end{array}$ & Rubiaceae & 65 & 5 & Lf. Fr. Wd. Bk. Rt & 0.253 & 2.43 & Guinko 661 (OUA) \\
\hline $\begin{array}{l}\text { Sclerocarya birrea (A. } \\
\text { Rich.) Hochst }\end{array}$ & Anacardiaceae & 31 & 8 & Lf. Fr. Wd. St. Bk. Ent & 0.12 & 2.43 & Ouédraogo. J. 15 (OUA) \\
\hline $\begin{array}{l}\text { Securidaca longipe- } \\
\text { dunculata Fresen }\end{array}$ & Polygalaceae & 52 & 6 & Lf. Fr. Wd. Bk. Rt. Ent & 0.21 & 2.43 & Guinko 392 (OUA) \\
\hline $\begin{array}{l}\text { Senegalia ataxa- } \\
\text { cantha (DC.) Kyal. \& } \\
\text { Boatwr }\end{array}$ & Fabaceae & 12 & 8 & Lf. Fr. Wd. Bk. Ent & 0.027 & 2.43 & $\begin{array}{l}\text { Tiné \& Bambara } 25 \\
\text { (OUA) }\end{array}$ \\
\hline $\begin{array}{l}\text { Senegalia gourmaen- } \\
\text { sis (A. Chev.) Kyal. \& } \\
\text { Boatwr }\end{array}$ & Fabaceae & 33 & 7 & Lf. Fr. Wd. Bk. Ent & 0.107 & 2.43 & $\begin{array}{l}\text { Ouédraogo. A. } 80 \\
\text { (OUA) }\end{array}$ \\
\hline $\begin{array}{l}\text { Senegalia macros- } \\
\text { tachya (Reichenb. ex } \\
\text { DC.) Kyal. \& Boatwr }\end{array}$ & Fabaceae & 12 & 5 & Lf. Fr. Wd. Ent & 0.05 & 2.43 & Madsen 5528 (OUA) \\
\hline $\begin{array}{l}\text { Senegalia polyacan- } \\
\text { tha (Willd.) Seigler \& } \\
\text { Ebinger }\end{array}$ & Fabaceae & 14 & 4 & Lf. Fr. Bk. Rt. Ent & 0.04 & 2.29 & $\begin{array}{l}\text { Ouédraogo. A. } 135 \\
\text { (OUA) }\end{array}$ \\
\hline $\begin{array}{l}\text { Senegalia senegal (L.) } \\
\text { Britton }\end{array}$ & Fabaceae & 1 & 1 & Lf & 0.333 & 1.86 & $\begin{array}{l}\text { Ouédraogo. A. } 136 \\
\text { (OUA) }\end{array}$ \\
\hline
\end{tabular}


Table 3 (continued)

\begin{tabular}{|c|c|c|c|c|c|c|c|}
\hline Plant taxon & Plant family & $\begin{array}{l}\text { Number of } \\
\text { reported } \\
\text { uses }\end{array}$ & $\begin{array}{l}\text { Number } \\
\text { of utilized } \\
\text { services }\end{array}$ & Used plant parts & Use Value & $\begin{array}{l}\text { Vulnerability } \\
\text { index }\end{array}$ & Voucher number \\
\hline $\begin{array}{l}\text { Sporobolus pyramida- } \\
\text { lis P.Beauv }\end{array}$ & Poaceae & 7 & 3 & St. Ent & 0.017 & 2 & Martinussen 55 (OUA) \\
\hline $\begin{array}{l}\text { Sterculia setigera } \\
\text { Delile }\end{array}$ & Malvaceae & 53 & 6 & Lf. Fr. Se. Bk. Rt. Ent & 0.217 & 2.43 & Thiombiano 858 (OUA) \\
\hline $\begin{array}{l}\text { Stereospermum kun- } \\
\text { thianum Cham }\end{array}$ & Bignoniaceae & 7 & 1 & Ent & 0.023 & 2.14 & $\begin{array}{l}\text { Ouédraogo. J. } 57 \\
\text { (OUA); }\end{array}$ \\
\hline $\begin{array}{l}\text { Striga hermonthica } \\
\text { (Delile) Benth }\end{array}$ & Orobanchaceae & 51 & 5 & Lf. Ent & 0.243 & 2.43 & Guinko 2323 (OUA) \\
\hline $\begin{array}{l}\text { Strophanthus hispidus } \\
\text { DC }\end{array}$ & Apocynaceae & 13 & 4 & Lf. Fr. Rt. Ent & 0.053 & 2.43 & $\begin{array}{l}\text { Thiombiano \& al. } 2693 \\
\text { (OUA) }\end{array}$ \\
\hline $\begin{array}{l}\text { Strychnos innocua } \\
\text { Delile }\end{array}$ & Loganiaceae & 5 & 3 & Lf. Rt. Ent & 0.019 & 2.29 & Guinko 114 (OUA); \\
\hline $\begin{array}{l}\text { Strychnos spinosa } \\
\text { Lam }\end{array}$ & Loganiaceae & 99 & 8 & Lf. Fr. Wd. Ent & 0.277 & 2.57 & Guinko 1360 (OUA) \\
\hline Tamarindus indica $\mathrm{L}$ & Fabaceae & 332 & 13 & $\begin{array}{l}\text { Lf. Fr. Se. Wd. Bk. Rt. } \\
\text { Ent }\end{array}$ & 0.99 & 2.57 & $\begin{array}{l}\text { Thiombiano } 1063 \\
\text { (OUA) }\end{array}$ \\
\hline Tapinanthus spp. & Loranthaceae & 27 & 6 & Lf. Fr. Wd. Ent & 0.117 & 2.29 & $\begin{array}{l}\text { Boussim } 10 \text { (OUA); } \\
\text { Boussim } 15 \text { (OUA); } \\
\text { Boussim } 16 \text { (OUA) }\end{array}$ \\
\hline $\begin{array}{l}\text { Tectona grandis L.f. } \\
\text { [cult.] }\end{array}$ & Lamiaceae & 112 & 9 & Lf. Fl. Wd.. Ent & 0.533 & 2.57 & $\begin{array}{l}\text { Schmidt \& al. } 1086 \\
\text { (OUA) }\end{array}$ \\
\hline $\begin{array}{l}\text { Tephrosia linearis } \\
\text { (Willd.) Pers }\end{array}$ & Fabaceae & 3 & 3 & Lf. Rt. Ent & 0.013 & 2 & Thiombiano 956 (OUA) \\
\hline $\begin{array}{l}\text { Terminalia avicen- } \\
\text { nioides Guill. \& Perr }\end{array}$ & Combretaceae & 45 & 9 & Lf. Wd. Bk. Rt. Ent & 0.16 & 2.43 & $\begin{array}{l}\text { Thiombiano } 613 \text { (OUA); } \\
\text { Thiombiano } 229 \text { (OUA); }\end{array}$ \\
\hline $\begin{array}{l}\text { Terminalia engleri } \\
\text { Gere \& Boatwr., }\end{array}$ & Combretaceae & 19 & 5 & Lf. Wd. Bk. Rt. Ent & 0.06 & 2.43 & Taïta 209 (OUA) \\
\hline $\begin{array}{l}\text { Terminalia macrop- } \\
\text { tera Guill. \& Perr }\end{array}$ & Combretaceae & 78 & 12 & Lf. Fr. Wd. Bk. Rt. Ent & 0.307 & 2.43 & Thiombiano 905 (OUA) \\
\hline $\begin{array}{l}\text { Terminalia schimperi } \\
\text { Hochst. ex Hutch. \& } \\
\text { Dalziel }\end{array}$ & Combretaceae & 148 & 12 & Lf. Fr. Wd. Bk. Rt. Ent & 0.593 & 2.57 & $\begin{array}{l}\text { Schmidt \& al. } 1186 \text { (FR; } \\
\text { OUA) }\end{array}$ \\
\hline Trichilia emetica Vahl & Meliaceae & 19 & 8 & Lf. Fr. St. Bk. Rt. Ent & 0.067 & 2.43 & Guinko 117 (OUA) \\
\hline $\begin{array}{l}\text { Triumfetta lepidota } \\
\text { K.Schum }\end{array}$ & Malvaceae & 13 & 2 & Ent & 0.043 & 2.14 & Guinko 1052 (OUA) \\
\hline $\begin{array}{l}\text { Vachellia nilotica (L.) } \\
\text { P.J.H.Hurter \& Mabb }\end{array}$ & Fabaceae & 66 & 8 & Lf. Fr. Bk. Rt. Ent & 0.303 & 2.43 & $\begin{array}{l}\text { Thiombiano } 3080 \\
\text { (OUA) }\end{array}$ \\
\hline $\begin{array}{l}\text { Vachellia seyal (Del.) } \\
\text { P.J.H.Hurter }\end{array}$ & Fabaceae & 3 & 2 & Lf. Rt & 0.01 & 2.29 & Guinko 851 (OUA) \\
\hline $\begin{array}{l}\text { Vachellia sieberiana } \\
\text { (DC.) Kyal. \& Boatwr }\end{array}$ & Fabaceae & 16 & 8 & Lf. Wd. Rt. Ent & 0.056 & 2.43 & Madsen 5111 (OUA) \\
\hline $\begin{array}{l}\text { Vernonia colorata } \\
\text { (Willd.) Drake }\end{array}$ & Asteraceae & 25 & 6 & Lf. Fr. Wd. Ent & 0.11 & 2.29 & $\begin{array}{l}\text { Thiombiano \& al. } 229 \\
\text { (OUA) }\end{array}$ \\
\hline $\begin{array}{l}\text { Vitellaria paradoxa } \\
\text { C.F. Gaertn }\end{array}$ & Sapotaceae & 960 & 14 & $\begin{array}{l}\text { Lf. Fr. Se. Wd. St. Bk. } \\
\text { Rt. Ent }\end{array}$ & 3.775 & 2.43 & Madsen 5171 (OUA) \\
\hline $\begin{array}{l}\text { Vitex chrysocarpa } \\
\text { Planch. ex Benth }\end{array}$ & Lamiaceae & 9 & 2 & Lf. Fr & 0.083 & 2.14 & Taïta 5 (OUA) \\
\hline Vitex doniana Sweet & Lamiaceae & 189 & 9 & Lf. Fr. Wd. Bk. Rt. Ent & 0.54 & 2.57 & Ouoba 175 (OUA) \\
\hline Ximenia americana L & Ximeniaceae & 165 & 5 & Lf. Fr. Wd. Bk. Rt. Ent & 0.653 & 2.57 & Guinko 1163 (OUA) \\
\hline $\begin{array}{l}\text { Zanthoxylum zan- } \\
\text { thoxyloides (Lam.) } \\
\text { Zepernick \&Timler }\end{array}$ & Rutaceae & 13 & 4 & Lf. Fr. Bk. Rt. Ent & 0.057 & 2.29 & Ouoba 48 (OUA) \\
\hline $\begin{array}{l}\text { Ziziphus mauritiana } \\
\text { Lam }\end{array}$ & Rhamnaceae & 19 & 3 & Lf. Rt. Ent & 0.072 & 2.29 & $\begin{array}{l}\text { Thiombiano \& al. } 199 \\
\text { (OUA) }\end{array}$ \\
\hline $\begin{array}{l}\text { Ziziphus mucronata } \\
\text { Willd }\end{array}$ & Rhamnaceae & 3 & 2 & Lf. Rt & 0.01 & 2.29 & $\begin{array}{l}\text { Guinko \& al. } 3373 \\
\text { (OUA) }\end{array}$ \\
\hline
\end{tabular}


Table 3 (continued)

Used plant parts: Lf. = Leaf; Fl. = Flower; Fr. = Fruit; Se. =Seed; Wd. =Wood; St. = stalk; Bk. = Bark; Rt. = Root; Ent $=$ Entire plant

Table 4 Variation in citation of ecosystem services provided by the ten most used species across the three land use intensity levels, the sociocultural groups, the gender, and age classes

\begin{tabular}{|c|c|c|c|c|c|c|c|c|c|c|c|c|c|}
\hline \multirow[t]{2}{*}{ ES categories } & \multirow[t]{2}{*}{ ES } & \multicolumn{3}{|c|}{ land use intensity level } & \multicolumn{3}{|c|}{ Sociocultural groups } & \multicolumn{3}{|c|}{ Gender } & \multicolumn{3}{|c|}{ Age classes } \\
\hline & & D.f & $x^{2}$ & $P$ value & D.f & $x^{2}$ & $P$ value & D.f & $x^{2}$ & $P$ value & D.f & $x^{2}$ & $P$ value \\
\hline Supporting & Soil fertility & 2 & 5.77 & 0.06 & 3 & 15.35 & 0.001537 & 1 & 14.59 & 0.0001 & 2 & 3.19 & 0.20 \\
\hline \multirow[t]{3}{*}{ Cultural } & Tourism & 2 & 47.11 & $<0.0001$ & 3 & 50.25 & $<0.0001$ & 1 & 1.02 & 0.31 & 2 & 3.37 & 0.18 \\
\hline & Religion & 2 & 60.16 & $<0.0001$ & 3 & 101.25 & $<0.0001$ & 1 & 0.14 & 0.71 & 2 & 0.43 & 0.81 \\
\hline & Ceremonies & 2 & 36.61 & $<0.0001$ & 3 & 76.85 & $<0.0001$ & 1 & 0.19 & 0.66 & 2 & 0.87 & 0.65 \\
\hline \multirow{5}{*}{ Regulating } & Water purification & 2 & 1.59 & 0.45 & 3 & 22.41 & $<0.0001$ & 1 & 1.48 & 0.22 & 2 & 3.49 & 0.17 \\
\hline & Shading & 2 & 50.37 & $<0.0001$ & 3 & 51.98 & $<0.0001$ & 1 & 0.22 & 0.64 & 2 & 1.30 & 0.52 \\
\hline & Wind protection & 2 & 42.20 & $<0.0001$ & 3 & 58.24 & $<0.0001$ & 1 & 0.34 & 0.56 & 2 & 0.19 & 0.91 \\
\hline & Pest control & 2 & 4.14 & 0.13 & 3 & 5.00 & 0.1716 & 1 & 12.52 & 0.0004 & 2 & 0.70 & 0.70 \\
\hline & Erosion prevention & 2 & 33.33 & $<0.0001$ & 3 & 31.59 & $<0.0001$ & 1 & 0.53 & 0.47 & 2 & 5.45 & 0.06 \\
\hline \multirow[t]{6}{*}{ Provisioning } & Medicinal use & 2 & 126.51 & $<0.0001$ & 3 & 142.52 & $<0.0001$ & 1 & 0.66 & 0.41 & 2 & 6.03 & 0.048 \\
\hline & Construction & 2 & 44.13 & $<0.0001$ & 3 & 52.04 & $<0.0001$ & 1 & 0.07 & 0.79 & 2 & 4.78 & 0.09 \\
\hline & Crafts & 2 & 61.24 & $<0.0001$ & 3 & 38.48 & $<0.0001$ & 1 & 6.29 & 0.01 & 2 & 5.33 & 0.07 \\
\hline & Energy supply & 2 & 14.38 & 0.0007 & 3 & 6.89 & $<0.0001$ & 1 & 0.38 & 0.53 & 2 & 0.50 & 0.78 \\
\hline & Fodder supply & 2 & 36.22 & $<0.0001$ & 3 & 24.11 & $<0.0001$ & 1 & 4.68 & 0.03 & 2 & 2.21 & 0.33 \\
\hline & Food supply & 2 & 61.18 & $<0.0001$ & 3 & 85.72 & $<0.0001$ & 1 & 7.26 & 0.007 & 2 & 5.82 & 0.054 \\
\hline
\end{tabular}

ES, ecosystem services

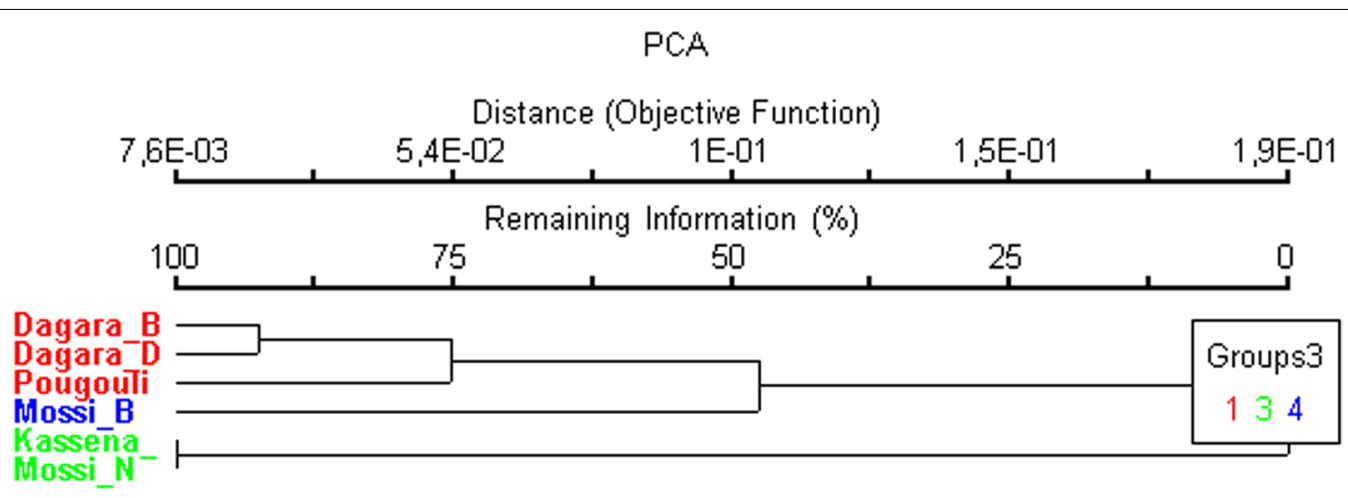

Fig. 5 Sociocultural group scores clustered according to similarity of citation of ecosystem services. Dagara_B: Dagara of TWRB (native); Dagara_D: Dagara of CAD (native); Mossi_N: Mossi of GRN (immigrant); Mossi_B: Mossi of TWRB (immigrant)

Local perceptions of availability and population dynamics of utilized species

The relative frequency of citation regarding the availability of all utilized species (Table 5) revealed that $60.12 \%$ of citations mentioned the abundant (45.86\%) to very abundant (14.24\%) of all utilized species. Among the most used species, Vitellaria paradoxa was most cited as very abundant species (35.04\%). In contrast, Adansonia digitata was most cited as rare species $(58.05 \%)$ followed by Faidherbia albida (55.20\%) which is only found in agrosystems.

The population dynamics of the species are generally conceived as static because the proportion of opinions in favor of a decrease in species (40.08\%) was approximately equal to that of the opinions in favor of their increase (40.86\%). However, only $19.06 \%$ stated a constancy (Table 5). Among the most used species, V. paradoxa is the species with the highest positive dynamics $(50 \%$ 


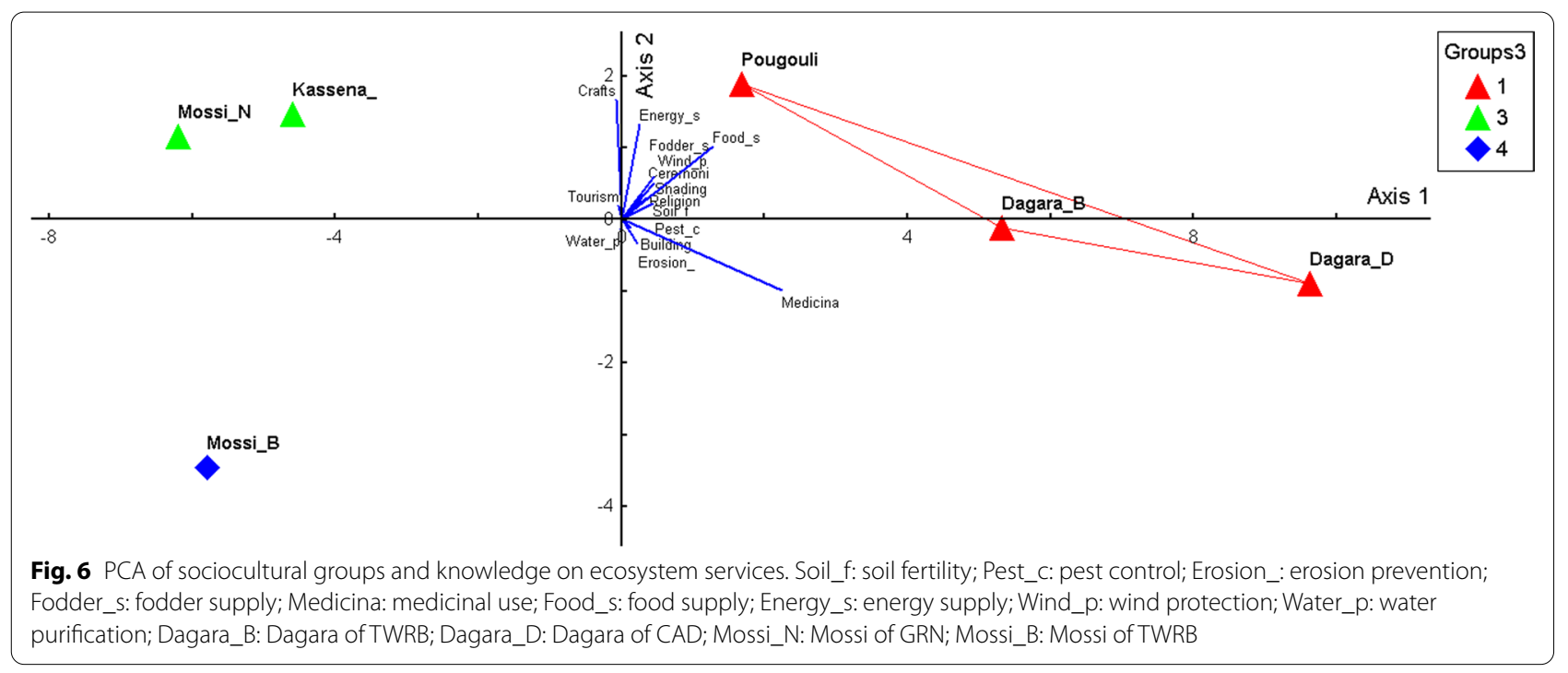

increase and $20.51 \%$ constancy) while $A$. digitata is the one with the highest negative dynamics ( $52.3 \%$ decrease).

\section{Perception of conservation of Sudanian savanna ecosystems by local populations}

Regarding the variables leading to vegetation degradation, local populations agree that fire, cutting of fresh wood, and clearing for extension of cultivated areas were most important. In terms of importance, the majority of the sample ranked fire as the first cause of ecosystem degradation. However, for the Dagara of TWRB, the Kassena, and the Mossi of GRN, demographic increase is the most important cause (Table 6).

Local populations ranked, by order of preference, five solutions for ecosystem conservation and four motivations for participating in sustainable management of ecosystems. For the suggested solutions, they ranked first "raising the awareness of local populations of the danger from degradation of natural resources," followed by "prohibition of fires by forest authorities" and "subsidy by the government" (Table 7). These solutions relate to a participation of local populations in the management of plant resources. The majority of the sample place awareness raising as the primary solution. However, the Pougouli, the Dagara of CAD, and the women place fire prohibition as the first solution, while the Dagara of TWRB place government subsidies as the first solution.

The motivation of local populations for sustainable management of plant resources is preferentially linked to their overall well-being due to the continuity of the provision of ES which will be ensured by long-term presence of the species, followed by the consideration of basic personal needs such as health, basic education, drinking water, and electricity provision by local authorities through the development of the village. Sustainability of vegetation is placed as the first motivation for sustainable management of plant resources by the majority of the sample. However, the elders, the Pougouli, the Mossi of GRN, and the men place village development as primary motivation (Table 8).

\section{Discussion}

\section{Diversity of utilized plant species and ecosystem services}

Altogether, 163 plant species were cited by local populations as those providing them with different ecosystem services from Sudanian savannas. When considering the total number of species (1410) found by Zizka et al. [10] in the South Sudanian phytogeographic sector of Burkina Faso, only $11.6 \%$ of the potential flora of the study area are used by local populations. Each ES involves a great diversity of plant species: at least 60 species are used for 10 ES. This diversity of used resources makes it possible to overcome the problem of insufficient plant resources for a given service and could be exploited for the substitution of the most threatened species by those having a sufficiently high abundance in vegetation [23]. However, some specific ES involve specific species with specific property and characteristic. For example, the construction service is provided by stable and resistant species such as Khaya senegalensis (Desv.) A. Juss., Anogeissus leiocarpa, Burkea africana Hook. and Pterocarpus erinaceus Poir. [5]. In the study area, the most cited services were food supply followed by medicinal services. The importance of both services has been reported from investigations in the West [40], South [41] and North [35] of Burkina Faso and Côte d'Ivoire [42].

The relatively high percentage of use of fruits and leaves could be explained by their importance in various 
Table 5 Relative frequency of perception of availability and dynamics of species utilized by local populations

\begin{tabular}{|c|c|c|c|c|c|c|}
\hline \multirow[t]{2}{*}{ Species } & \multicolumn{3}{|c|}{ Species availability (\%) } & \multicolumn{3}{|c|}{ Species dynamics (\%) } \\
\hline & Rare & Abundant & Very abundant & Decrease & Constancy & Increase \\
\hline All species & 39.88 & 45.86 & 14.24 & 40.08 & 19.05 & 40.86 \\
\hline Acacia spp (ex) & 50.00 & 50.00 & 0.00 & 33.33 & 50.00 & 16.67 \\
\hline Adansonia digitata $\mathrm{L}$ & 58.05 & 29.89 & 12.07 & 52.30 & 19.54 & 28.16 \\
\hline Afzelia africana Sm. ex Pers & 38.25 & 54.10 & 7.65 & 40.44 & 17.49 & 42.08 \\
\hline Agave sisalana Perrine [cult.] & 45.45 & 36.36 & 18.18 & 36.36 & 18.18 & 45.45 \\
\hline Anacardium occidentale L. [cult.] & 53.33 & 26.67 & 20.00 & 33.33 & 26.67 & 40.00 \\
\hline Andropogon chinensis (Nees) Merr & 4.35 & 71.74 & 23.91 & 15.22 & 2.17 & 82.61 \\
\hline Andropogon gayanus Kunth & 10.53 & 37.72 & 51.75 & 7.89 & 6.14 & 85.96 \\
\hline Andropogon spp & 52.94 & 29.41 & 17.65 & 11.76 & 47.06 & 41.18 \\
\hline Annona senegalensis Pers & 37.50 & 52.08 & 10.42 & 45.83 & 12.50 & 41.67 \\
\hline Azadirachta indica A.Juss. [cult.] & 50.48 & 43.81 & 5.71 & 36.19 & 23.81 & 40.00 \\
\hline Balanites aegyptiaca (L.) Delile & 39.51 & 54.32 & 6.17 & 46.91 & 16.05 & 37.04 \\
\hline Boerhavia diffusa $\mathrm{L}$ & 22.22 & 77.78 & 0.00 & 44.44 & 11.11 & 44.44 \\
\hline Bombax costatum Pellegr. \& Vuill & 60.00 & 35.38 & 4.62 & 75.38 & 4.62 & 20.00 \\
\hline Bridelia scleroneura Müll. Arg & 100.00 & 0.00 & 0.00 & 71.43 & 0.00 & 28.57 \\
\hline Burkea africana Hook & 53.57 & 35.71 & 7.14 & 42.86 & 25.00 & 28.57 \\
\hline Cadaba farinosa Forssk & 0.00 & 100.00 & 0.00 & 50.00 & 0.00 & 50.00 \\
\hline Calotropis procera (Ait.) Ait. f & 37.50 & 62.50 & 0.00 & 50.00 & 12.50 & 37.50 \\
\hline Capparis sepiaria $\mathrm{L}$ & 66.67 & 11.11 & 22.22 & 66.67 & 0.00 & 33.33 \\
\hline Capsicum frutescens L. [cult.] & 0.00 & 57.14 & 42.86 & 0.00 & 0.00 & 100.00 \\
\hline Carica papaya L. [cult.] & 84.62 & 12.82 & 2.56 & 56.41 & 17.95 & 25.64 \\
\hline Cassia nigricans Vahl & 49.09 & 18.18 & 32.73 & 34.55 & 5.45 & 60.00 \\
\hline Cassia obtusifolia L & 23.08 & 38.46 & 38.46 & 7.69 & 7.69 & 84.62 \\
\hline Cassia sieberiana DC & 60.00 & 40.00 & 0.00 & 68.00 & 20.00 & 12.00 \\
\hline Ceiba pentandra (L.) Gaertn & 71.43 & 25.00 & 3.57 & 50.00 & 21.43 & 28.57 \\
\hline Chasmopodium caudatum (Hack.) Stapf & 22.22 & 44.44 & 33.33 & 33.33 & 5.56 & 61.11 \\
\hline Chrysopogon nigritanus (Benth.) Veldkamp & 100.00 & 0.00 & 0.00 & 100.00 & 0.00 & 0.00 \\
\hline Citrus aurantium L. [cult.] & 41.18 & 47.06 & 11.76 & 29.41 & 35.29 & 35.29 \\
\hline Citrus limon (L.) Burm.f. [cult.] & 73.81 & 23.81 & 2.38 & 38.10 & 28.57 & 33.33 \\
\hline Cochlospermum planchonii Hook. f. ex Planch & 66.67 & 33.33 & 0.00 & 66.67 & 33.33 & 0.00 \\
\hline Cola cordifolia (Cav.) R.Br & 42.86 & 57.14 & 0.00 & 42.86 & 57.14 & 0.00 \\
\hline Cola laurifolia Mast & 33.33 & 33.33 & 33.33 & 33.33 & 0.00 & 66.67 \\
\hline Coldenia procumbens $\mathrm{L}$ & 50.00 & 50.00 & 0.00 & 0.00 & 0.00 & 100.00 \\
\hline Combretum adenogonium Steud. ex A. Rich & 47.62 & 52.38 & 0.00 & 47.62 & 28.57 & 23.81 \\
\hline Combretum collinum Fresen & 45.65 & 47.83 & 6.52 & 43.48 & 39.13 & 17.39 \\
\hline Combretum glutinosum Perr. ex DC & 0.00 & 100.00 & 0.00 & 100.00 & 0.00 & 0.00 \\
\hline Combretum molle G. Don & 66.67 & 33.33 & 0.00 & 66.67 & 0.00 & 33.33 \\
\hline Combretum nigricans Lepr. ex Guill. \& Perr & 100.00 & 0.00 & 0.00 & 100.00 & 0.00 & 0.00 \\
\hline Combretum paniculatum Vent & 62.50 & 37.50 & 0.00 & 62.50 & 0.00 & 37.50 \\
\hline Combretum sericeum G. Don & 40.00 & 60.00 & 0.00 & 80.00 & 0.00 & 20.00 \\
\hline Corchorus olitorius L & 29.41 & 52.94 & 17.65 & 35.29 & 5.88 & 58.82 \\
\hline Cordia myxa L & 40.26 & 53.25 & 6.49 & 33.77 & 37.66 & 28.57 \\
\hline Crateva adansonii DC & 63.64 & 24.68 & 11.69 & 46.75 & 19.48 & 33.77 \\
\hline Crossopteryx febrifuga (Afzel. ex G.Don) Benth & 30.61 & 67.35 & 2.04 & 53.06 & 16.33 & 30.61 \\
\hline Cyanotis lanata Benth & 80.00 & 20.00 & 0.00 & 0.00 & 40.00 & 60.00 \\
\hline Cymbopogon caesius (Nees ex Hook. \& Arn.) Stapf & 10.71 & 50.00 & 39.29 & 25.00 & 0.00 & 75.00 \\
\hline Cymbopogon schoenanthus (L.) Spreng & 30.43 & 43.48 & 26.09 & 52.17 & 13.04 & 34.78 \\
\hline Dalbergiaboehmii Taub & 0.00 & 100.00 & 0.00 & 0.00 & 0.00 & 100.00 \\
\hline
\end{tabular}


Table 5 (continued)

\begin{tabular}{|c|c|c|c|c|c|c|}
\hline \multirow[t]{2}{*}{ Species } & \multicolumn{3}{|c|}{ Species availability (\%) } & \multicolumn{3}{|c|}{ Species dynamics (\%) } \\
\hline & Rare & Abundant & Very abundant & Decrease & Constancy & Increase \\
\hline Dalbergia melanoxylon Guill. \& Perr & 0.00 & 100.00 & 0.00 & 100.00 & 0.00 & 0.00 \\
\hline Daniellia oliveri (Rolfe) Hutch. \& Dalziel & 26.15 & 66.15 & 7.69 & 35.38 & 20.00 & 46.15 \\
\hline Detarium microcarpum Guill. \& Perr & 32.03 & 54.90 & 13.07 & 35.95 & 18.95 & 45.10 \\
\hline Dichrostachys cinerea (L.) Wight \& Arn & 41.67 & 50.00 & 8.33 & 58.33 & 16.67 & 25.00 \\
\hline Dicoma tomentosa Cass & 0.00 & 0.00 & 100.00 & 0.00 & 0.00 & 100.00 \\
\hline Diospyros mespiliformis Hochst. ex A. DC & 23.70 & 65.90 & 10.40 & 38.15 & 23.12 & 38.73 \\
\hline Echinochloa pyramidalis (Lam.) Hitchc. \& Chase & 11.11 & 77.78 & 11.11 & 27.78 & 0.00 & 72.22 \\
\hline Entada africana Guill. \& Perr & 64.29 & 32.14 & 3.57 & 28.57 & 21.43 & 50.00 \\
\hline Erythrina senegalensis A.DC & 50.00 & 50.00 & 0.00 & 25.00 & 25.00 & 50.00 \\
\hline Eucalyptus camaldulensis Dehnh. [cult.] & 63.64 & 30.30 & 6.06 & 33.33 & 18.18 & 48.48 \\
\hline Fadogia agrestis Schweinf. ex Hiern & 0.00 & 100.00 & 0.00 & 0.00 & 0.00 & 100.00 \\
\hline Faidherbia albida (Delile) A.Chev. Del & 55.20 & 39.20 & 5.60 & 45.60 & 24.80 & 29.60 \\
\hline Feretia apodanthera Delile & 22.22 & 66.67 & 11.11 & 22.22 & 33.33 & 44.44 \\
\hline Ficus abutilifolia (Miq.) Miq & 0.00 & 77.78 & 22.22 & 33.33 & 22.22 & 44.44 \\
\hline Ficus dicranostyla Mildbr & 87.50 & 0.00 & 12.50 & 100.00 & 0.00 & 0.00 \\
\hline Ficus ingens (Miq.) Miq & 0.00 & 100.00 & 0.00 & 100.00 & 0.00 & 0.00 \\
\hline Ficus platyphylla Delile & 41.67 & 50.00 & 8.33 & 33.33 & 16.67 & 50.00 \\
\hline Ficus sur Forssk & 50.00 & 50.00 & 0.00 & 100.00 & 0.00 & 0.00 \\
\hline Ficus sycomorus $L$ & 33.09 & 54.68 & 12.23 & 38.85 & 21.58 & 39.57 \\
\hline Flacourtia indica (Burm. f.) Merrill & 66.67 & 33.33 & 0.00 & 0.00 & 100.00 & 0.00 \\
\hline Flueggea virosa (Roxb. ex Willd.) Voigt & 33.33 & 50.00 & 16.67 & 66.67 & 16.67 & 16.67 \\
\hline Gardenia erubescens Stapf \& Hutch & 39.25 & 51.40 & 9.35 & 43.93 & 19.63 & 36.45 \\
\hline Gardenia ternifolia Schumach. \& Thonn & 100.00 & 0.00 & 0.00 & 100.00 & 0.00 & 0.00 \\
\hline Gmelina arborea Roxb. [cult.] & 62.50 & 37.50 & 0.00 & 62.50 & 25.00 & 12.50 \\
\hline Grewia bicolor Juss & 50.00 & 50.00 & 0.00 & 50.00 & 0.00 & 50.00 \\
\hline Grewia cissoides Hutch. \& Dalziel & 0.00 & 100.00 & 0.00 & 0.00 & 0.00 & 100.00 \\
\hline Grewia lasiodiscus K.Schum & 20.00 & 40.00 & 40.00 & 80.00 & 0.00 & 20.00 \\
\hline Grewia spp & 0.00 & 100.00 & 0.00 & 100.00 & 0.00 & 0.00 \\
\hline Guiera senegalensis J.F.Gmel & 19.05 & 76.19 & 4.76 & 38.10 & 0.00 & 61.90 \\
\hline Gymnosporia senegalensis (Lam.) Loes & 69.23 & 15.38 & 15.38 & 53.85 & 7.69 & 38.46 \\
\hline Haematostaphis barteri Hook.f & 25.00 & 75.00 & 0.00 & 25.00 & 0.00 & 75.00 \\
\hline Hibiscus cannabinus L. [cult.] & 14.29 & 28.57 & 57.14 & 57.14 & 0.00 & 42.86 \\
\hline Hymenocardia acida Tul & 22.22 & 77.78 & 0.00 & 66.67 & 11.11 & 22.22 \\
\hline Hyparrhenia spp & 50.00 & 50.00 & 0.00 & 50.00 & 0.00 & 50.00 \\
\hline Hyptis spicigera Lam & 22.22 & 51.39 & 26.39 & 6.94 & 33.33 & 59.72 \\
\hline Indigofera bracteolata DC & 100.00 & 0.00 & 0.00 & 50.00 & 0.00 & 50.00 \\
\hline Ipomoea carnea Jacq & 0.00 & 100.00 & 0.00 & 50.00 & 0.00 & 50.00 \\
\hline Isoberlinia doka Craib \& Stapf & 37.25 & 54.90 & 7.84 & 41.18 & 7.84 & 50.98 \\
\hline Jatropha curcas L. [cult.] & 66.67 & 18.52 & 14.81 & 7.41 & 11.11 & 81.48 \\
\hline Khaya senegalensis (Desv.) A. Juss & 29.24 & 49.12 & 21.64 & 45.61 & 13.45 & 40.94 \\
\hline Kigelia africana (Lam.) Benth & 50.00 & 50.00 & 0.00 & 80.00 & 10.00 & 10.00 \\
\hline Landolphia heudelotii A. DC & 71.43 & 28.57 & 0.00 & 28.57 & 42.86 & 28.57 \\
\hline Lannea acida A. Rich & 54.29 & 31.43 & 14.29 & 54.29 & 17.14 & 28.57 \\
\hline Lannea microcarpa Engl. \& K. Krause & 25.53 & 60.64 & 13.83 & 34.57 & 23.94 & 40.96 \\
\hline Lannea velutina A.Rich & 0.00 & 100.00 & 0.00 & 0.00 & 100.00 & 0.00 \\
\hline Leptadenia hastata (Pers.) Decne & 50.00 & 37.50 & 12.50 & 62.50 & 12.50 & 25.00 \\
\hline Loeseneriella africana (Willd.) N.Hallé & 0.00 & 100.00 & 0.00 & 0.00 & 0.00 & 100.00 \\
\hline Lophira lanceolata Tiegh. ex Keay & 68.89 & 28.89 & 2.22 & 42.22 & 15.56 & 42.22 \\
\hline
\end{tabular}


Table 5 (continued)

\begin{tabular}{|c|c|c|c|c|c|c|}
\hline \multirow[t]{2}{*}{ Species } & \multicolumn{3}{|c|}{ Species availability (\%) } & \multicolumn{3}{|c|}{ Species dynamics (\%) } \\
\hline & Rare & Abundant & Very abundant & Decrease & Constancy & Increase \\
\hline Loudetia simplex (Nees) C.E.Hubb & 0.00 & 0.00 & 100.00 & 0.00 & 0.00 & 100.00 \\
\hline Loudetia togoensis (Pilg.) C.E.Hubb & 0.00 & 0.00 & 100.00 & 0.00 & 0.00 & 100.00 \\
\hline Maerua angolensis DC & 83.33 & 16.67 & 0.00 & 83.33 & 16.67 & 0.00 \\
\hline Maerua crassifolia Forssk & 100.00 & 0.00 & 0.00 & 100.00 & 0.00 & 0.00 \\
\hline Mangifera indica L. [cult.] & 68.03 & 24.59 & 7.38 & 45.08 & 23.77 & 31.15 \\
\hline Mimosa pigra L & 16.67 & 50.00 & 33.33 & 16.67 & 0.00 & 83.33 \\
\hline Mitragyna inermis (Willd.) Kuntze & 63.33 & 33.33 & 3.33 & 56.67 & 23.33 & 20.00 \\
\hline Moringa oleifera $\mathrm{L}$ & 70.00 & 24.29 & 5.71 & 50.00 & 20.00 & 30.00 \\
\hline Nymphaea lotus $L$ & 57.14 & 14.29 & 28.57 & 57.14 & 0.00 & 42.86 \\
\hline Ocimum americanum $\mathrm{L}$ & 8.70 & 34.78 & 56.52 & 10.87 & 6.52 & 82.61 \\
\hline Oncoba spinosa Forssk & 50.00 & 33.33 & 16.67 & 50.00 & 38.89 & 11.11 \\
\hline Opilia amentacea Roxb & 46.67 & 33.33 & 20.00 & 53.33 & 13.33 & 33.33 \\
\hline Oxytenanthera abyssinica (A.Rich.) Munro & 100.00 & 0.00 & 0.00 & 100.00 & 0.00 & 0.00 \\
\hline Ozoroa obovata (Oliv.) R.Fern. \& A.Fern & 0.00 & 100.00 & 0.00 & 100.00 & 0.00 & 0.00 \\
\hline Parinari curatellifolia Planch. ex Benth & 50.00 & 50.00 & 0.00 & 100.00 & 0.00 & 0.00 \\
\hline Parkia biglobosa (Jacq.) R. Br. ex G. Don f & 29.33 & 50.96 & 19.71 & 41.35 & 16.83 & 41.83 \\
\hline Paullinia pinnata $\mathrm{L}$ & 28.57 & 42.86 & 28.57 & 28.57 & 14.29 & 57.14 \\
\hline Pennisetum pedicellatum Trin & 50.00 & 0.00 & 50.00 & 25.00 & 0.00 & 75.00 \\
\hline Pennisetum spp & 12.00 & 28.00 & 60.00 & 24.00 & 4.00 & 72.00 \\
\hline Pericopsis laxiflora (Benth. ex Bak.) van Meeuwen & 20.00 & 73.33 & 6.67 & 33.33 & 6.67 & 60.00 \\
\hline Philenoptera laxiflora (Guill. \& Perr.) Roberty & 38.89 & 61.11 & 0.00 & 22.22 & 0.00 & 77.78 \\
\hline Piliostigma reticulatum (DC.) Hochst & 56.52 & 17.39 & 26.09 & 60.87 & 17.39 & 26.09 \\
\hline Piliostigma thonningii (Schum.) Milne-Redhead & 44.12 & 35.29 & 20.59 & 42.65 & 20.59 & 36.76 \\
\hline Prosopis africana (Guill. \& Perr.) Taub & 50.00 & 40.91 & 9.09 & 31.82 & 36.36 & 31.82 \\
\hline Pseudocedrela kotschyi (Schweinf.) Harms & 21.74 & 56.52 & 21.74 & 30.43 & 17.39 & 52.17 \\
\hline Psidium guajava L. [cult.] & 100.00 & 0.00 & 0.00 & 61.90 & 14.29 & 23.81 \\
\hline Pterocarpus erinaceus Poir & 32.32 & 56.10 & 11.59 & 43.90 & 21.95 & 34.15 \\
\hline Pterocarpus santalinoides DC & 100.00 & 0.00 & 0.00 & 100.00 & 0.00 & 0.00 \\
\hline Raphionacme splendens Schltr & 50.00 & 50.00 & 0.00 & 0.00 & 0.00 & 100.00 \\
\hline Rottboellia cochinchinensis (Lour.) Clayton & 28.33 & 45.00 & 26.67 & 5.00 & 51.67 & 43.33 \\
\hline Rytigynia senegalensis Blume & 0.00 & 0.00 & 100.00 & 0.00 & 0.00 & 100.00 \\
\hline Saba senegalensis (A. DC.) Pichon & 30.22 & 58.27 & 11.51 & 33.09 & 29.50 & 36.69 \\
\hline Sarcocephalus latifolius (Sm.) E.A.Bruce & 39.47 & 39.47 & 21.05 & 57.89 & 5.26 & 36.84 \\
\hline Sclerocarya birrea (A. Rich.) Hochst & 76.19 & 23.81 & 0.00 & 57.14 & 14.29 & 28.57 \\
\hline Securidaca longipedunculata Fresen & 65.85 & 26.83 & 7.32 & 70.73 & 7.32 & 21.95 \\
\hline Senegalia ataxacantha (DC.) Kyal. \& Boatwr & 16.67 & 50.00 & 33.33 & 16.67 & 33.33 & 50.00 \\
\hline Senegalia gourmaensis (A.Chev.) Kyal. \& Boatwr & 4.76 & 66.67 & 28.57 & 33.33 & 4.76 & 61.90 \\
\hline Senegalia macrostachya (Reichenb. ex DC.) Kyal. \& Boatwr & 30.00 & 30.00 & 40.00 & 60.00 & 0.00 & 40.00 \\
\hline Senegalia polyacantha (Willd.) Seigler \& Ebinger & 57.14 & 42.86 & 0.00 & 14.29 & 28.57 & 57.14 \\
\hline Senegalia senegal (L.) Britton & 0.00 & 100.00 & 0.00 & 0.00 & 0.00 & 100.00 \\
\hline Sporobolus pyramidalis P.Beauv & 33.33 & 50.00 & 16.67 & 50.00 & 0.00 & 50.00 \\
\hline Sterculia setigera Delile & 64.86 & 29.73 & 5.41 & 48.65 & 18.92 & 32.43 \\
\hline Stereospermum kunthianum Cham & 28.57 & 42.86 & 28.57 & 42.86 & 0.00 & 57.14 \\
\hline Striga hermonthica (Delile) Benth & 41.18 & 41.18 & 17.65 & 0.00 & 72.55 & 27.45 \\
\hline Strophanthus hispidus DC & 57.14 & 42.86 & 0.00 & 57.14 & 0.00 & 42.86 \\
\hline Strychnos innocua Delile & 75.00 & 25.00 & 0.00 & 75.00 & 25.00 & 0.00 \\
\hline Strychnos spinosa Lam & 21.54 & 64.62 & 13.85 & 41.54 & 10.77 & 47.69 \\
\hline Tamarindus indica $\mathrm{L}$ & 31.40 & 54.55 & 14.05 & 47.93 & 14.05 & 38.02 \\
\hline
\end{tabular}


Table 5 (continued)

\begin{tabular}{|c|c|c|c|c|c|c|}
\hline \multirow[t]{2}{*}{ Species } & \multicolumn{3}{|c|}{ Species availability (\%) } & \multicolumn{3}{|c|}{ Species dynamics (\%) } \\
\hline & Rare & Abundant & Very abundant & Decrease & Constancy & Increase \\
\hline Tapinanthus spp & 68.42 & 31.58 & 0.00 & 31.58 & 5.26 & 63.16 \\
\hline Tectona grandis L.f. [cult.] & 72.00 & 28.00 & 0.00 & 26.00 & 28.00 & 46.00 \\
\hline Tephrosia linearis (Willd.) Pers & 33.33 & 0.00 & 66.67 & 0.00 & 33.33 & 66.67 \\
\hline Terminalia avicennioides Guill. \& Perr & 29.03 & 61.29 & 9.68 & 70.97 & 9.68 & 19.35 \\
\hline Terminalia engleri Gere \& Boatwr. & 46.67 & 33.33 & 20.00 & 46.67 & 20.00 & 33.33 \\
\hline Terminalia macroptera Guill. \& Perr & 58.97 & 41.03 & 0.00 & 41.03 & 35.90 & 23.08 \\
\hline $\begin{array}{l}\text { Terminalia schimperi Hochst. ex Hutch. \& } \\
\text { Dalziel }\end{array}$ & 29.63 & 55.56 & 14.81 & 38.27 & 22.22 & 39.51 \\
\hline Trichilia emetica Vahl & 66.67 & 25.00 & 8.33 & 41.67 & 0.00 & 58.33 \\
\hline Triumfetta lepidota K.Schum & 30.77 & 61.54 & 7.69 & 46.15 & 0.00 & 53.85 \\
\hline Vachellia nilotica (L.) P.J.H.Hurter \& Mabb & 84.21 & 13.16 & 2.63 & 52.63 & 21.05 & 26.32 \\
\hline Vachellia seyal (Del.) P.J.H.Hurter & 0.00 & 100.00 & 0.00 & 0.00 & 0.00 & 100.00 \\
\hline Vachellia sieberiana (DC.) Kyal. \& Boatwr & 50.00 & 28.57 & 21.43 & 35.71 & 14.29 & 50.00 \\
\hline Vernonia colorata (Willd.) Drake & 62.50 & 31.25 & 6.25 & 50.00 & 6.25 & 43.75 \\
\hline Vitellaria paradoxa C.F. Gaertn & 18.80 & 46.15 & 35.04 & 29.49 & 20.51 & 50.00 \\
\hline Vitex chrysocarpa Planch. ex Benth & 87.50 & 12.50 & 0.00 & 50.00 & 25.00 & 25.00 \\
\hline Vitex doniana Sweet & 31.07 & 57.28 & 11.65 & 42.72 & 10.68 & 46.60 \\
\hline Ximenia americana $\mathrm{L}$ & 43.96 & 52.75 & 3.30 & 46.15 & 14.29 & 39.56 \\
\hline Zanthoxylum zanthoxyloides (Lam.) Zepernick \& Timler & 45.45 & 54.55 & 0.00 & 45.45 & 9.09 & 45.45 \\
\hline Ziziphus mauritiana Lam & 41.67 & 50.00 & 8.33 & 75.00 & 8.33 & 16.67 \\
\hline Ziziphus mucronata Willd & 0.00 & 100.00 & 0.00 & 100.00 & 0.00 & 0.00 \\
\hline
\end{tabular}

Table 6 Average score and rank of causes of ecosystem degradation among different groups in the sample

\begin{tabular}{|c|c|c|c|c|c|c|c|}
\hline Informants & Fire & Wood cutting & Clearing & $\begin{array}{l}\text { Demographic } \\
\text { increase }\end{array}$ & Pasture & Climate change & $\begin{array}{l}\text { Forest } \\
\text { management }\end{array}$ \\
\hline Global & 5.04 & 4.88 & 4.73 & 4.48 & 3.48 & 3.21 & 2.13 \\
\hline \multicolumn{8}{|l|}{ Age classes } \\
\hline Young & 5.20 & 4.83 & 4.89 & 4.33 & 3.72 & 3.17 & 1.84 \\
\hline Adult & 5.09 & 4.92 & 4.43 & 4.52 & 3.28 & 3.48 & 2.18 \\
\hline Elders & 4.66 & 4.91 & 5.04 & 4.68 & 3.43 & 2.75 & 2.53 \\
\hline \multicolumn{8}{|c|}{ Sociocultural groups } \\
\hline Dagara_B & 4.92 & 4.30 & 4.68 & 5.02 & 3.60 & 2.80 & 2.32 \\
\hline Dagara_D & 5.70 & 5.48 & 4.80 & 2.76 & 3.28 & 3.06 & 2.92 \\
\hline Pougouli & 5.05 & 4.75 & 4.80 & 4.70 & 2.90 & 2.90 & 2.90 \\
\hline Mossi_B & 5.35 & 5.20 & 4.85 & 4.60 & 2.50 & 3.40 & 2.10 \\
\hline Mossi_N & 4.74 & 4.64 & 4.60 & 4.94 & 3.72 & 3.72 & 1.64 \\
\hline Kassena & 4.66 & 5.04 & 4.78 & 5.08 & 3.92 & 3.30 & 1.36 \\
\hline \multicolumn{8}{|l|}{ Gender } \\
\hline Man & 4.94 & 5.01 & 4.85 & 4.45 & 3.40 & 3.43 & 2.19 \\
\hline Women & 5.13 & 4.76 & 4.62 & 4.52 & 3.55 & 2.98 & 2.08 \\
\hline
\end{tabular}

Emphasis: Bold—1st rank, italic_2nd rank, bold italics_3rd rank; Sociocultural groups: Dagara_B: Dagara of TWRB; Dagara_D: Dagara of CAD; Mossi_N: Mossi of GRN; Mossi_B: Mossi of TWRB

services such as food, fodder supply and medicinal use. The high frequency of fruit citation $(70 \%)$ for food supply shows the importance of fruit in the diet of local populations. Wild fruits contribute to a varied diet in terms of vitamin (A, B, C, D, and E) and micronutrient intake $[41,43]$. For example, the content of vitamin $C$ in 
Table 7 Average score and rank of suggested solutions for ecosystem conservation among different groups in the sample

\begin{tabular}{|c|c|c|c|c|c|}
\hline Informants & $\begin{array}{l}\text { Awareness of } \\
\text { degradation }\end{array}$ & Fire prohibition & $\begin{array}{l}\text { Subsidy from the } \\
\text { government }\end{array}$ & $\begin{array}{l}\text { Inspection and } \\
\text { reforestation }\end{array}$ & $\begin{array}{l}\text { Inclusive } \\
\text { management }\end{array}$ \\
\hline Global & 2.27 & 1.75 & 1.42 & 1.28 & 0.95 \\
\hline \multicolumn{6}{|l|}{ Age classes } \\
\hline Young & 2.25 & 1.98 & 1.40 & 1.08 & 0.80 \\
\hline Adult & 2.43 & 1.62 & 1.65 & 1.16 & 0.94 \\
\hline Elders & 2.00 & 1.64 & 1.04 & 1.81 & 1.23 \\
\hline \multicolumn{6}{|c|}{ Sociocultural groups } \\
\hline Dagara_B & 2.40 & 0.92 & 2.78 & 1.46 & 1.20 \\
\hline Dagara_D & 2.84 & 3.52 & 0.80 & 1.30 & 0.26 \\
\hline Pougouli & 2.25 & 2.45 & 0.30 & 1.55 & 1.40 \\
\hline Mossi_B & 2.05 & 1.00 & 1.60 & 0.75 & 0.50 \\
\hline Mossi_N & 1.92 & 1.40 & 1.14 & 1.04 & 0.76 \\
\hline Kassena & 2.02 & 1.20 & 1.34 & 1.40 & 1.58 \\
\hline \multicolumn{6}{|l|}{ Gender } \\
\hline Men & 2.44 & 1.20 & 1.64 & 1.33 & 1.28 \\
\hline Women & 2.10 & 2.31 & 1.20 & 1.22 & 0.62 \\
\hline
\end{tabular}

Emphasis: Bold-1st rank, italic_2nd rank, bold italics—3rd rank; Sociocultural groups: Dagara_B: Dagara of TWRB; Dagara_D: Dagara of CAD; Mossi_N: Mossi of GRN; Mossi_B: Mossi of TWRB

Table 8 Average score and rank of motivations for ecosystem conservation among different groups in the sample

\begin{tabular}{|c|c|c|c|c|}
\hline Informants & Vegetation sustainability & Village development & Diversification of income & Obtaining a Job \\
\hline Global & 2.96 & 2.93 & 2.49 & 1.65 \\
\hline \multicolumn{5}{|l|}{ Age classes } \\
\hline Young & 3.03 & 3.02 & 2.44 & 1.59 \\
\hline Adult & 2.86 & 2.75 & 2.61 & 1.76 \\
\hline Elders & 3.02 & 3.11 & 2.34 & 1.53 \\
\hline \multicolumn{5}{|c|}{ Sociocultural groups } \\
\hline Dagara_B & 3.00 & 2.80 & 2.14 & 2.06 \\
\hline Dagara_D & 2.96 & 2.56 & 2.68 & 1.80 \\
\hline Pougouli & 2.65 & 3.10 & 2.50 & 1.75 \\
\hline Mossi_B & 3.10 & 2.75 & 2.80 & 1.20 \\
\hline Mossi_N & 2.90 & 3.36 & 2.52 & 1.38 \\
\hline Kassena & 3.04 & 3.00 & 2.48 & 1.48 \\
\hline \multicolumn{5}{|l|}{ Gender } \\
\hline Men & 2.88 & 3.00 & 2.35 & 1.89 \\
\hline Women & 3.03 & 2.86 & 2.63 & 1.40 \\
\hline
\end{tabular}

Emphasis: Bold_-1st rank, italic_2nd rank, bold italics_3rd rank; Sociocultural groups; Dagara_B: Dagara of TWRB; Dagara_D: Dagara of CAD; Mossi_N: Mossi of GRN; Mossi_B: Mossi of TWRB

fruit of Adansonia digitata and Detarium microcarpa is as high as in orange fruit; Moringa oleifera contains twice as much protein as yogurt. In addition, plant species used for food supply were also used for medicinal service. Sourabié et al. [44] reported anti-diarrheal effects of the fruit's pulp of Adansonia digitata and lowering of hypertension by Parkia biglobosa seeds.
The highest relative frequency (31\%) of citations of the whole plant shows that local people are aware of the importance of vegetation and trees for their well-being, as the services they associate with the whole plant are regulatory, cultural, and supporting. These services are not destructive for plants and ecosystems but are rather conservative. This demonstrates local populations being 
committed to the conservation of their environment which constitutes their living space.

The 10 species with the highest use values were all woody species, and the shea tree (Vitellaria paradoxa) is a very popular species with the highest use value (3.775). In addition to its use in almost all services (14 of 15), it has a real use (UV) far more important than that of other species and a high socioeconomic value. This oleaginous species represents the fourth exportation product of Burkina Faso after gold, cotton, and livestock. Its high importance for populations was confirmed by other ethnobotanical studies [6, 24, 40,41, 45, 46]. Almonds and fruits of Vitellaria paradoxa, fruit pulp and seeds of Parkia biglobosa, and fruit pulp and leaves of Adansonia digitata are highly appreciated as non-timber forest products which provide income through their trade [47]. Shea butter (from Vitellaria paradoxa) and the African mustard, also called soumbala (from Parkia biglobosa), are transformed products with strong chains of added values [48, 49]. Diospyros mespiliformis and Lannea microcarpa have highly appreciated edible fruits and medicinal uses. Faidherbia albida is a fodder woody species most appreciated by livestock breeders because it bears leaves and pods during the dry season when most of the woody species have shed leaves and fruits [35]. Faidherbia albida also has a high value of fertilizing cultivated or fallow soils [33] and is seen as a mystic plant by the Mossi [50]. Khaya senegalensis, Ficus sycomorus, Afzelia africana and Pterocarpus erinaceus are also fodder species with medicinal and cultural value $[20,50,51]$. Anti-malarial effects of Afzelia africana, Khaya senegalensis, Ficus sycomorus, Parkia biglobosa and Pterocarpus erinaceus are reported from Ghana [52]. The medicinal uses of the species most cited from our study area are also mentioned from other regions of Burkina Faso [35, 44, 53].

With regard to the high use value and the high demand for the products of most cited species, they constitute key species for local populations according to the definition by Clark and Sunderland [54]. However, once the value of a NTFP and the intensity of its use are extremely high, the resource is very likely to be overexploited, causing it to become locally extinct [54]. Gaisberger et al. [55] showed that overexploitation of species has emerged as the most important short-term threat. Overexploitation is the single most important threat for Parkia biglobosa (41.2\%) and Vitellaria paradoxa (41.1\%), and is only slightly exceeded by climate change in the case of Adansonia digitata (38.0\%). The ethno-botanical use values correlated with the number of uses identified for each species and revealed the species preferred by local populations. However, the results must be taken with caution as the applied method does not distinguish between past, present and potential uses (some species may disappear because of anthropogenic pressure) $[45,56]$.

\section{Variables influencing the knowledge of ecosystem services} The three levels of land use intensity of this study design account for the economic benefit that local communities derive from them. The populations of the CAD and the TWRB live mainly from agriculture. The populations living near the TWRB engage in illegal activities such as farming, pasture and wood cutting in the protected area [14] as they do not benefit from economic benefits of the protected area in the same way as those of GRN. In fact, at least $18 \%$ of TWRB had been cleared [57] by local populations to install their fields. Forest administration has great difficulties to prevent neighboring villagers from using the resources in protected zones that they highly depend on $[7,13]$ as long as no incentives are offered for compensation. In contrast, inhabitants living near GRN are employed with the forest officers to ensure functioning of this tourist attraction and vary their income by participating in forest management. Likewise, hunting in the village hunting zone around GRN and fishing in water points of the ranch provide populations with additional income. Therefore, providing biodiversity conservation actors with diversified sources of income is economically important to local communities $[15,58]$. The management of natural resources involving local communities contributes to better security of biodiversity through sustainable participatory management [59].

As for the sociocultural groups, the Pougouli and Dagara had more knowledge in ES referring to food supply, religion, wind protection, shading, medicinal use, soil fertilization, erosion prevention and energy supply. Their knowledge in religion services expresses the animist cult influence of Pougouli. According to the national statistics of population, the populations of the Southwest region have $64.9 \%$ of animists [60]. The high rate of religion ES is a means to preserve the surrounding vegetation and ecosystem. Religions are excellent channels for transmitting local knowledge through initiations (i.e., traditional and spiritual instructions) which are part of the education of the youngest in the preservation of the environmental values. To the animist communities such as Pougouli and Dagara, forests are the habitats of venerate spirits [50, 51]. The good knowledge about species providing medicinal services may be explained by the preservation of their ancestral knowledge transmitted from generation to generation $[3,16,61]$. The use of species for food supply, wind protection, shading and energy supply ES reflects the poverty of these populations who heavily depend on income from agriculture and small livestock. The Dagara and Pougouli are introvert sociocultural groups little open to exterior influence and quite 
attached to their local environment. Pougouli and Dagara knowledge is also orientated to performing farming. They are essentially farmers and do not hesitate to transgress protected areas to install their fields [14]. The population of GRN pays little attention to medicinal services due to the fact that they live nearby a health center, leading to a loss of local knowledge about medicinal plants. Local populations are subjected to increasing social (demographic and economic) and environmental pressures which have mostly led to a loss of knowledge [24, 62]. TWRB Mossi had no specific knowledge about ES provided by Sudanian savanna species. The lack of specific knowledge about Sudanian vegetation of the Mossi migrants of TWRB could be related to the fact of having immigrated from the Sudano-Sahelian zone where the species composition is different. The ethnobotanical knowledge varies, effectively, across sociocultural groups because of cultural differences and social habits $[4,63]$. In addition, the migrant communities of TWRB are located outside the natives' villages which constitutes an obstacle for passing on and sharing inter-community knowledge.

On the contrary, the Kassena and GRN Mossi sociocultural groups possessed similar knowledge and had more knowledge in craft, energy and touristic services than the Mossi of TWRB, the Native of TWRB and CAD. This demonstrates that the Mossi migrants of GRN are well integrated and adapted to the native way of life. The Mossi migrants of GRN have replaced species which they originally used by species which serve the same purpose among the natives (the Kassena). Thus, the traditional use of species by migrant communities can be influenced more strongly by the environment than by cultural heritage [64].

\section{Vulnerability of the species}

The multipurpose use of species, the utilization of slow regenerating plant organs (as wood, seeds, bark, roots and flowers), and the preference (the high use value) that populations have for some species act to increase their vulnerability. The 10 species with high use value were highly vulnerable, except for Faidherbia albida and Vitellaria paradoxa classified as moderately vulnerable. The state of vulnerability of Adansonia digitata, Afzelia africana, Khaya senegalensis, Parkia biglobosa and Pterocarpus erinaceus is confirmed by Thiombiano et al. [65] who classify them threatened species of the southern Sudanian zone. According to these authors, Diospyros mespiliformis would be more threatened in the northern Sudanian zone. In the sub-Sahelian zone, Ouedraogo et al. [35] confirm high vulnerability of Adansonia digitata, Diospyros mespiliformis, Faidherbia albida, Khaya senegalensis, Lannea microcarpa, Parkia biglobosa, Pterocarpus erinaceus and Vitellaria paradoxa. All the 10 most used species of this study are also considered endangered by local populations of northern Benin [66]. Nevertheless, for the vast majority of plant species in Burkina Faso insufficient data are available for a full IUCN assessment $[11,27]$. Globally and according to the IUCN red list [67], the conservation status of Pterocarpus erinaceus has been decreasing and has become Endangered, indicating a high risk of extinction. Afzelia africana, Khaya senegalensis and Vitellaria paradoxa conservation status are classified Vulnerable, indicating risk of extinction. The status of Parkia biglobosa, Diospyros mespiliformis, Adansonia digitata, Lannea microcarpa, Faidherbia albida and Ficus sycomorus is Least Concern. Although these species have different levels of vulnerability in other regions of Burkina Faso $[11,35]$ due to utilization by local populations, they are of great interest to the populations of our study sites. It would then be appropriate to think about their sustainable management through rational use motivated by the perceptions of local populations.

\section{Local perceptions about availability and population dynamics of the most used species}

Perceptions of local populations on the availability and population dynamics of used species follow the same trend in the study area. Local populations stated that Vitellaria paradoxa was the most abundant species and showed an increase, whereas Adansonia digitata was very rare and showed a decrease. Traoré et al. [20] found that, in the Southwest of the country, perception of the state of resources by the local Senufo is consistent with the findings in the field. Indeed, $64 \%$ of the species cited by the Senufo as being rare are part of the rare species revealed by the calculation of the rarity index at the end of floristic inventories. Thus, the least variation in the availability of important species is perceived by local populations. The socioeconomic importance, availability and population dynamics of species define the management of traditional agroforestry systems. According to Assogbadjo et al. [21], species perceived by local communities as threatened are integrated into traditional agroforestry systems. However, the populations admit general degradation of the ecosystems.

\section{Local perception of conservation of Sudanian savanna ecosystems}

According to local populations, fire, deforestation and clearing constitute the three main causes of Sudanian vegetation degradation. Local populations use fire as a tool for hunting, clearing of village surroundings and field preparation [63]. Deforestation by wood cutting results from the demand for energy, craft and construction services. The clearing of natural vegetation is practiced for installing new and extending existing agricultural land. 
Local populations are conscious of the causes of the degradation of plant resources, being essentially them provoking ecological pressure originating from increasing needs of growing populations $[7,13,14]$. While the development of agriculture has made it possible to free oneself from dependence on wild food, the expansion of agricultural land strongly contributes to ecosystem and biodiversity degradation [68]. Land use changes effectively have a negative impact on biodiversity due to habitat loss or fragmentation [20]. In the area of high land use intensity (CAD), species diversity is low and only species which provide ES are conserved.

Raising the awareness of local populations in the face of degrading natural resources is the first solution unanimously suggested by the communities. Given that climate change is not well perceived at the local scale, awareness raising may contribute to reducing anthropogenic pressure (fire, wood logging). Local population's awakening of awareness as to the vulnerability of plant resources used every day and their implication for natural resources management in collaboration with forest authorities are steps that would enhance sustainable conservation of plants. The success of biodiversity conservation efforts often depends on local populations, especially when these communities are the key players in ecosystem management [69]. In fact, high biodiversity existing in native territories around the world is the result of traditional knowledge and management practices [16]. Fair collaboration of local populations with the forest authority for conservation of plant resources could be achieved, for example, by meeting certain basic needs such as the creation of water reservoirs and the construction of health centers and schools. Sustainability of vegetation allows for diversification of incomes, especially for women who collect and sell NTFP to complete their incomes [24]. Local processing of NTFP by women before selling contributes to the value chain of products and generating incomes. For example, the almond of Vitellaria paradoxa is transformed into shea butter and the seed of Parkia biglobosa is transformed into soumbala (African mustard) $[48,49]$.

\section{Conclusion}

This study has highlighted the importance of ES of Sudanian savannas and the importance of long-term preservation of numerous plant species. It shows that local populations do know their environment well and that they are aware of the causes of degradation of plant resources. Therefore, local people should be involved in programs and projects addressing sustainable management and conservation of Sudanian savanna ecosystems. The behavioral change can be achieved by awareness raising and education. Education of local populations needs to involve best management strategies and promotion of domestication and regeneration of local multipurpose species. Also species with low utilization value should be protected for conserving future biological resources because species being less important today could be sought for in the future to replace others having become rare in the meantime.

However, in face of local population vulnerability and their strong dependence on natural resources, it is often difficult to consolidate conservation and rational and sustainable use of biodiversity. In order to achieve effective awareness raising among local populations for sustainable management of plant resources, it would be necessary to offer them solutions and motivations adapted to their perceptions and their consents. This approach will enable full collaboration of the sociocultural communities.

\section{Abbreviations}

ES: Ecosystem services; TWRB: Total wildlife reserve of Bontioli (medium land use intensity); CAD: Communal area of Dano (high land use intensity); GRN: Game ranch of Nazinga (low land use intensity.

\section{Acknowledgements}

The authors thank the West Africa Science Service Center on Climate Change and Adapted Land Use (WASCAL) program funded by the German Federal Ministry for Education and Research (BMBF) and the National Center for Information, Scholar and Professional Orientation, and Scholarship (CIOSPB) for the financial support. Many thanks to the National Office of Protected Areas (OFINAP) to have given us access to protected areas, and especially to local populations of communal area of Dano (CAD), total wildlife reserve of Bontioli (TWRB), and game ranch of Nazinga (GRN) for their cooperation and the sharing of their knowledge.

\section{Authors' contributions}

AN and DG conceived the work with advices from AT. AN collected the data. AN processed the data and performed the statistical analyses. AN and DG drafted the manuscript with contribution of AO, SP, and AT. AT supervised the work. All authors read and approved the final manuscript.

\section{Funding}

This research was supported by the BMBF (German Federal Ministry of Education and Research) through the program WASCAL (West African Science

Service Center on Climate Change and Adapted Land Use,www.wascal.org).

Availability of data and materials

The datasets used and/or analyzed in the current study are available from the corresponding author on reasonable request.

\section{Declarations}

Ethics approval and consent to participate

Individual consent to participate in the study was obtained prior to the study by the administration of the questionnaire. Only people that consented to participate in the study were considered.

Consent for publication

Not applicable.

Competing interests

The authors declare that they have no competing interests. 


\section{Author details}

${ }^{1}$ Laboratory of Plant Biology and Ecology, University Joseph Ki-Zerbo, 03 BP 7021 Ouagadougou 03, Burkina Faso. ${ }^{2}$ Institute of Biological Sciences, Department of Botany, University of Rostock, 18051 Rostock, Germany.

Received: 16 August 2021 Accepted: 5 February 2022 Published online: 19 February 2022

\section{References}

1. Millennium Ecosystem Assessment (MEA). Ecosystem wealth and human well-being. Washington: Island Press; 2005

2. UICN France. Panorama des services écologiques fournis par les milieux naturels en France-volume 1 : contexte et enjeux. Paris, France, 2012.

3. Bognoumou F, Savadogo P, Thiombiano A, Boussim Jl, Oden PC, Guinko S. Informants based ethnobotany and utility evaluation of five combretaceae species: differentiation by socioculturality and geographical location. Forests Trees Livelihoods. 2011;20:265-82.

4. Sop TK, Oldeland J, Schmiedel U, Thiombiano A. Ethnobotanical knowledge and valuation of woody plants species: a comparative analysis of three sociocultural groups from the sub-Sahel of Burkina Faso. Environ Dev Sustain. 2012. https://doi.org/10.1007/s10668-012-9345.-9.

5. Ouédraogo I, Nacoulma BMl, Hahn K, Thiombiano A. Assessing ecosystem services based on indigenous knowledge in south-eastern Burkina Faso (West Africa). Int J Biodivers Sci Ecosyst Serv Manag. 2014;10(4):313-21.

6. Zizka A, Thiombiano A, Dressler S, Nacoulma BI, Ouédraogo A Ouédraogo I, Ouédraogo O, Zizka G, Hahn K, Schmidt M. Traditional plant use in Burkina Faso (West Africa): a national-scale analysis with focus on traditional medicine. J Ethnobiol Ethnomed. 2015;11:9. https://doi.org/10. 1186/1746-4269-11-9.

7. Belem B, Nacoulma BMI, Gbangou R, Kambou S, Hansen HH, Gausset Q, Lund S, Raebild A, Lompo D, Ouedraogo M, Theilade I, Boussim IJ. Use of non wood forest products by local people bordering the "Parc National Kaboré Tambi." J Transdiscipl Environ Stud. 2007;6(1):1-21.

8. Ngom D, Charahabil MM, Sarr O, Bakhoum A, Akpo LE. Perceptions communautaires sur les services écosystémiques d'approvisionnement fournis par le peuplement ligneux de la Réserve de Biosphère du Ferlo (Sénégal). VertigO. 2014. https://doi.org/10.4000/vertigo.15188.

9. Fontès J, Guinko S. Carte de la végétation et de l'occupation du sol du Burkina Faso. Notice explicative. Ministère de la Coopération française, projet Campus, Toulouse. 1995: 68.

10. Zizka A, Thiombiano A, Dressler S, Nacoulma BMl, Ouédraogo A, Ouédraogo I, Ouédraogo O, Zizka G, Hahn K, Schmidt M. The vascular plant diversity of Burkina Faso (West Africa) — a quantitative analysis and implications for conservation. Candollea. 2015;70(1):9-20.

11. Schmidt M, Zizka A, Traoré S, Ataholo M, Chatelain C, Daget P, Dressler S, Hahn K, Kirchmair I, Krohmer J, Mbayngone E, Müller JV, Nacoulma B, Ouédraogo A, Ouédraogo O, Sambaré O, Schumann K, Wieringa JJ, Zizka $\mathrm{G}$, Thiombiano A. Diversity, distribution and preliminary conservation status of the flora of Burkina Faso. Phytotaxa. 2017;304(1):1-215. https:// doi.org/10.11646/phytotaxa.304.1.1.

12. Institut National de la Statistique et de la Demographie (INSD). Résultats préliminaires du cinquième recensement général de la population et de I'habitat du Burkina Faso. 2020.

13. Gnoumou A, Rusu E, Adouabou B, Thiombiano A. Spatial and temporal dynamics of the vegetation of Comoé-léraba reserve and its surrounding lands (Burkina Faso, West Africa). PESD. 2016. https://doi.org/10.1515/ pesd-2016-0002.

14. Dimobe K, Ouédraogo A, Soma S, Goetze D, Porembski S, Thiombiano A. Identification of driving factors of land degradation and deforestation in the Wildlife Reserve of Bontioli (Burkina Faso, West Africa). Global Ecol Conserv. 2015;4:559-71.

15. Dimobe K, Goetze D, Ouédraogo A, Forkuor G, Wala K, Porembski S, Thiombiano A. Spatio-temporal dynamics in land use and habitat fragmentation within a protected area dedicated to tourism in a Sudanian Savanna of West Africa. J Landsc Ecol. 2017;10(1):75-95.

16. Diegues AC. The role of ethnoscience in the build-up of ethnoconservation as a new approach to nature conservation in the tropics. The case of
Brazil. Revue d'ethnoécologie. 2014. https://doi.org/10.4000/ethnoecolo gie.1956.

17. OCDE (Organization of Cooperation and Economic Development). Climate change and agriculture: impacts, adaptation, mitigation and options for the OECD [COM/TAD/CA/ENV/EPOC. 2009;13]. http://olisweb. oecd.org/. Accessed 26 Sept 2019.

18. Bambara D, Bilgo A, Hien E, Masse D, Thiombiano A, Hien V. Influencedes composts de dechets urbains sur les rendements du Sorgho en zone soudano-sahélienne du Burkina Faso. Revue Ivoirienne de Sciences et Technologie. 2014;24:148-71.

19. Holou RAY, Sinsin B. Embroussaillements des pâturages artificiels et naturels exploités par les bovins en zone guinéennes au Bénin. Ann Sci Agron Bénin. 2002;3(1):40-66.

20. Traore L, Ouedraogo I, Ouedraogo A, Thiombiano A. Perceptions, usages et vulnérabilité des ressources végétales ligneuses dans le Sud-Ouest du Burkina Faso. Int J Biol Chem Sci. 2011;5(1):258-78.

21. Assogbadjo AE, Glèlè Kakaï R, Vodouhê FG, Djagoun CAMS, Codjia JTC, Sinsin B. Biodiversity and socioeconomic factors supporting farmers' choice of wild edible trees in the agroforestry systems of Benin (West Africa). Forest Policy Econ. 2012;14:41-9.

22. Kaboré SA, Hien M, Ouédraogo D, Diallo TRE, Hahn K, Nacro BH. Use of ecosystem services of Sarcocephalus latifolius (Sm.) E. A. Bruce and induced effect of human pressure on the species in the Southwestern Region of Burkina Faso. Ethnobot Res Appl. 2014;12:561-70.

23. Kaboré SA, Schumann K, Hien M, Lykke AM, Hahn K, Nacro BH. Stratégies d'adaptation à la réduction des services écosystémiques : cas des Potentialités de substitution de trois espèces forestières dans le Sud-Ouest du Burkina Faso. Int J Biol Chem Sci. 2015;9(3):1194-208.

24. Tiétiambou FRS, Lykke AM, Korbéogo G, Thiombiano A, Ouédraogo A. Perceptions et savoirs locaux sur les espèces oléagineuses locales dans le Kénédougou, Burkina Faso. Bois et Forets des Tropiques. 2016;327(1):39-50.

25. Dimobe K, Tondoh JE, Weber JC, Bayala J, Ouédraogo K, Greenough K. Farmers' preferred tree species and their potential carbon stocks in southern Burkina Faso: implications for biocarbon initiatives. PLOS ONE. 2018;13(12): e0199488. https://doi.org/10.1371/journal.pone.0199488.

26. Stein K, Stenchly K, Coulibaly D, Pauly A, Dimobe K, Steffan-Dewenter I, Konaté S, Goetze D, Porembski S, Linsenmair KE. Impact of human disturbance on bee pollinator communities in savanna and agricultural sites in Burkina Faso, West Africa. Ecol Evol. 2018;8:6827-38. https://doi.org/10. 1002/ece3.4197.

27. Nacoulma BMI, Ouédraogo I, Ouédraogo O, Dimobe K, Thiombiano A. Phytodiversity of Burkina Faso: Selected Countries in Africa. In: Global Biodiversity, Pullaiah T, editors. Apple Academic Press Inc. 2018; 3: 1-33. ISBN 978-0-42946-980-0

28. Houéhanou DT, Assogbadjo AE, Chadare FJ, Zanvo S, Sinsin B. Approches méthodologiques synthétisées des études d'ethnobotanique quantitative en milieu tropical. Annales des Sciences Agronomiques. 2016; 20 - spécial Projet Undesert-UE : ISSN 1659-5009. pp 187-205

29. Albuquerque UP, Ramos MA, Lucena RFP, Alencar NL. Methods and techniques used to collect ethnobiological data. Springer Protocols Handbooks. 2014. https://doi.org/10.1007/978-1-4614-8636-7_2.

30. Berhaut J. Flore du Sénégal. Edition Clairafrique, Dakar. 1967: 485.

31. LeBourgeois T, Merlier H. Advantrop. Les adventices d'Afrique soudanosahélienne. Montpellier, France, CIRAD-CA éditeurs. 1995: 640.

32. Poilecot P. Les Poaceae du Niger. Description - Illustration - Ecologie - Utilisations. Boissiera. Genève, Suisse, 1999; 56: 766. ISBN CIRAD 2-87614-342-9.

33. Arbonnier M. Arbres, arbustes et lianes d'Afrique de l'Ouest. Ed. Quae. 2019: 776.

34. Betti JL. Vulnérabilité des plantes utilisées comme antipaludiques dans l'arrondissement de Mintom au sud de la réserve de Biosphère du Dja (Cameroun). Syst Geogr Plant. 2001;71:661-78.

35. Ouédraogo P, Bationo BA, Sanou J, Traoré S, Barry S, Dayamba SD, Bayala J, Ouédraogo M, Soeters S, Thiombiano A. Uses and vulnerability of ligneous species exploited by local population of northern Burkina Faso in their adaptation strategies to changing environments. Agric Food Secur. 2017:6:15.

36. Thiombiano A, Glele Kakaï R, Bayen P, Boussim JI, Mahamane A. Méthodes et dispositifs d'inventaires forestiers en Afrique de l'Ouest : état des lieux et propositions pour une harmonisation. Annales des Sciences 
Agronomiques. 2016; 20 - spécial Projet Undesert-UE: 15-31 (2016) ISSN 1659-5009.

37. Camou-Guerrero A, Reyes-García V, Martínez-Ramos M, Casas A. Knowledge and use value of plant species in a rarámuri community: a gender perspective for conservation. Hum Ecol. 2008;36:259-72.

38. R Core Team. R: A language and environment for statistical computing. R Foundation for Statistical Computing, Vienna, Austria. 2019. https:// www.R-project.org/.

39. Lawrence A, Phillips OL, Ismodes AR, Lopez M, Rose S, Wood D, Farfan AJ. Local values for harvested forest plants in Madre de Dios, Peru: towards a more contextualised interpretation of quantitative ethnobotanical data. Biodivers Conserv. 2005;14:45-79.

40. Taita P. Use of woody plants by locals in Mare aux Hippopotames Biosphere Reserve in western Burkina. Biodivers Conserv. 2003;12:1203-17.

41. Guigma Y, Zerbo P, Ouédraogo A, Millogo-rasolodimby J. The use of wild plants as food in three adjoining villages in Southern Burkina Faso. J Nutr Ecol Food Res. 2014;2:1-11.

42. Vroh B, Ouattara $D$, Kpangui $K$. Disponibilité des espèces végétales spontanées à usage traditionnel dans la localité d'Agbaou, Centre-ouest de la Côte d'Ivoire. J Appl Biosci. 2014;76(1):6386-96.

43. Boedecker J, Termote C, Assogbadjo AE, Van Damme P, Lachat C. Dietary contribution of Wild Edible Plants to women's diets in the buffer zone around the Lama forest, Benin — an underutilized potential. Food Sec. 2014;6:833-49. https://doi.org/10.1007/s12571-014-0396-7.

44. Sourabie TS, Kinda D, Yaro B, Nikiema JB. Ethnobotanical survey of medicinal plants used by the traditional medical healers in the villages of Bérégadougou and Fabédougou (Cascades Region, Burkina Faso). IOSR J Pharmacy. 2013;3(7):38-45.

45. Bélem B, Smith Olsen C, Theilade I, Bellefontaine R, Guinko S, Mette Lykke A, Diallo A, Boussim IJ. Identification des arbres hors forêt préférés des populations du Sanmatenga (Burkina Faso). Bois et Forêts des Tropiques. 2008:298(4):53-64.

46. Cissé M, Bationo BA, Traoré S, Boussim IJ. Perception d'espèces agroforestières et de leurs services écosystémiques par trois groupes ethniques du bassin versant de Boura, zone soudanienne du Burkina Faso. Bois and Forets Des Tropiques. 2019;338:29-42.

47. Ouédraogo M, Ouédraogo D, Thiombiano T, Hien M, Lykke AM. Dépendance économique aux produits forestiers non ligneux: cas des ménages riverains des forêts de Boulon et de Koflandé, au Sud-Ouest du Burkina Faso. J Agric Environ Int Dev. 2013:107(1):45-72.

48. Badini Z, Kaboré M, Mheen-Sluijer J, Vellema S. Chaînes de valeur de la filière karité au Burkina Faso. Wageningen UR/Agriterra/AgriProFocus. 2011;14:40.

49. Kronborg M. Improving livelihoods in West Africa through a natural resources-The case of Parkia biglobosa and Soumbala. PhD thesis. Aarhus University. 2015.

50. Savadogo S, Sop TK, Thiombiano A. Sacred and totemic plants among thirty two sociocultural groups in burkina faso: implications for biodiversity conservation. Annales des sciences agronomiques. 2017;21(1):89-120.

51. Balima LH, Nacoulma BMI, Ekué MRM, Kouame FN, Thiombiano A. Use patterns, use values and management of Afzelia africana Sm in Burkina Faso: implications for species domestication and sustainable conservation. J Ethnobiol Ethnomed. 2018;14(23):1-14.

52. Asase A, Oteng-yeboah AA, Odamtten GT, Simmonds MSJ. Ethnobotanical study of some Ghanaian anti-malarial plants. J Ethnopharmacol. 2005;99:273-9.

53. Zerbo P, Millogo-Rasolodimby J, Nacoulma-Ouedraogo O, Van Damme P. Plantes médicinales et pratiques médicales au Burkina Faso: cas des Sanan. Bois Et Forêts Des Tropiques. 2011;307(1):41-53.

54. Clark EL, Sunderland TC. The key non-timber Forest Products of central Africa: state of the Knowledge. Technical paper $\mathrm{N}^{\circ} 122$. SD publication Series. Office of sustainable Development Bureau for Africa. USAID. 2004. http://hdl.handle.net/10919/65996

55. Gaisberger H, Kindt R, Loo J, Schmidt M, Bognounou F, Da SS, et al. Spatially explicit multi-threat assessment of food tree species in Burkina Faso: A fine-scale approach. PLoS ONE. 2017;12(9): e0184457. https://doi.org/ 10.1371/journal.pone.0184457.

56. Zenderland Y, Hart R, Bussmann RW, Paniagua Zambrana NY, Sikharulidze S, Kikvidze Z, Kikodze D, Tchelidze D, Khutsishvili M, Batsatsashvili K. The use of "use value": quantifying importance in ethnobotany. Econ Bot. 2019. https://doi.org/10.1007/s12231-019-09480-1.

57. UICN/PACO. Évaluation de l'efficacité de la gestion des aires protégées: aires protégées du Burkina Faso. 2009.

58. Bouché P, Lungren CG, Hien B. Recensement aérien total de la faune dans I'Écosystème naturel Po-Nazinga-Sissili (PONASI). Burkina Faso. Programme CITES-MIKE. 2004 ; 85

59. Ouédraogo M. Régulation de la dynamique des populations de buffles (Syncerus caffer Sparrman) et de Waterbucks (Kobus ellipsiprymnus Ogilby) et moyens de gestion à mettre en oeuvre pour préserver l'équilibre des communautés végétales dans le ranch de Nazinga (Burkina Faso). Unpublished Ph.D. Thesis, University of Gembloux, Belgique. 2005.

60. Institut National de la Statistique et de la Demographie (INSD). Annuaire statistique 2017 de la région du Sud Ouest. 2018.

61. Lykke AM. Local perceptions of vegetation change and priorities for conservation of woody-savanna vegetation in Senegal. J Environ Manag. 2000:59:107-20. https://doi.org/10.1006/jema.2000.0336.

62. Albuquerque UP, Nascimento ALB, Soldati GT, Feitosa IS, Campos JLA, Hurrell JA, Hanazaki N, Medeiros PM, Silva RRV, Ludwinsky RH, Ferreira WSJ, Reyes-García V. Ten important questions/issues for ethnobotanical research. Acta Botanica Brasilica. 2019;33(2):376-85. https://doi.org/10. 1590/0102-33062018abb0331.

63. Koulibaly A, Traoré D, Goetze D. Cacao and plant diversity. In: Atlas de la Biodiversité de I'Afrique de I'Ouest, Tome II: Burkina Faso, Thiombiano A and Kampmann D, editors. Ouagadougou and Frankfurt/Main, 2010.

64. Medeiros PM, Soldati GT, Alencar NL, Vandebroek I, Pieroni A, Hanazaki $\mathrm{N}$, Albuquerque UP. The use of medicinal plants by migrant people: adaptation, maintenance and replacement. Review article. Evid-Based Complement Altern Med. 2012. https://doi.org/10.1155/2012/807452.

65. Thiombiano A, Schmidt M, Da S, Hahn-Hadjali K, Zizka G, Wittig R. Vascular plants: flowering plants. In: Thiombiano A, Kampmann D, editors. Atlas de la Biodiversité de l'Afrique de l'Ouest, Tome II: Burkina Faso. Ouagadougou and Frankfurt/Main, 2010.

66. Agbani PO, Kafoutchoni KM, Salako KV, Gbedomon RC, Kégbé AM, Karen H, Sinsin B. Traditional ecological knowledge-based assessment of threatened woody species and their potential substitutes in the Atakora mountain chain, a threatened hotspot of biodiversity in Northwestern Benin, West Africa. J Ethnobiol Ethnomed. 2018;14(1):1-19.

67. IUCN. Red list. https://www.iucnredlist.org. Accessed 25 Nov 2020.

68. Mbayngone E, Thiombiano A. Dégradation des aires protégées par l'exploitation des ressources végétales : cas de la réserve partielle de faune de Pama, Burkina Faso (Afrique de l'Ouest). Fruits. 2011;66(3):187-202.

69. Jones ET, Lynch KA. Non timber forest products and biodiversity management in the Pacific Northwest. For Ecol Manag. 2007;246:29-37.

\section{Publisher's Note}

Springer Nature remains neutral with regard to jurisdictional claims in published maps and institutional affiliations.

$$
\begin{aligned}
& \text { Ready to submit your research? Choose BMC and benefit from: } \\
& \text { - fast, convenient online submission } \\
& \text { - thorough peer review by experienced researchers in your field } \\
& \text { - rapid publication on acceptance } \\
& \text { - support for research data, including large and complex data types } \\
& \text { - gold Open Access which fosters wider collaboration and increased citations } \\
& \text { - maximum visibility for your research: over } 100 \mathrm{M} \text { website views per year }
\end{aligned}
$$

\section{At $\mathrm{BMC}$, research is always in progress.}

Learn more biomedcentral.com/submissions 